\title{
Exploración de las características psicométricas del inventario de estrés ocupacional - OSI para el contexto colombiano
}

\author{
Exploration of the psychometric characteristics of the inventory \\ of occupational stress - OSI for the Colombian context
}

ISSN 0124-0137 EISNN 2027-212X
Artículo de Investigación

Copyright 2018 by Psicogente

Correspondencia de autores:

mgarcia@ucatolica.edu.co

hjvelezb@unal.edu.co

\author{
Mónica García Rubiano y Helena Vélez Botero \\ Universidad Católica de Colombia, Bogotá, Colombia
}

Resumen

Objetivo: Brindar una herramienta en el contexto colombiano que permita evaluar el estrés desde una perspectiva de afrontamiento, se busca explorar las características psicométricas del inventario de estrés ocupacional-OSI.

\begin{abstract}
Método:El diseño utilizado fue transversal de tipo descriptivo - correlacional. Para este fin, respondieron el cuestionario 500 adultos trabajadores (hombres y mujeres) de empresas públicas y privadas de Colombia, con edades entre los 17 y 71 años. Se analizaron las propiedades psicométricas relacionadas con la dificultad y discriminación de los ítems a través de la prueba t, la confiabilidad a través del coeficiente de Cronbach y la validez factorial mediante un análisis de máxima verosimilitud con rotación varimax
\end{abstract}

Resultados: Indican que el inventario de estrés ocupacional-OSI posee alta confiabilidad ( $\alpha=0.88$ ) y homogeneidad parcial en las escalas y subescalas que componen el instrumento, las cuales explican el $43 \%$ de la varianza total en el comportamiento del estrés ocupacional.

Conclusiones: El inventario de estrés ocupacional-OSI es una herramienta potencialmente útil para evaluar el estrés en población colombiana.

\author{
Palabras Claves: \\ Estrés ocupacional, Afrontamiento, Validez, Confiabilidad.
}

\section{Abstract}

Objective: To provide a tool that allows stress evaluation from a coping perspective to explore the psychometric characteristics of the occupational stress-OSI in Colombian context.

\begin{abstract}
Method: A descriptive cross - sectional design - correlational. For exploring the psychometric characteristics of the occupational stress-OSI, 500 adult male workers, between 17 and 71 years of age from public and private companies in Colombia were sampled through a questionnaire. The psychometric properties related to the difficulty and discrimination of the items through the T- test, the reliability through the Cronbach coefficient and the factorial validity through a maximum likelihood analysis with varimax rotation.
\end{abstract}

Results: The occupational stress-OSI inventory has high reliability $(\alpha=0.88)$ and partial homogeneity in the scales and subscales that make up the instrument, which explain $43 \%$ of the total variance in the behavior of occupational stress.

Conclusion: The occupational stress inventory-OSI is a potentially useful tool to evaluate stress in the Colombian population.

Key words:Occupational stress, Coping, Validity, Reliability.

Como citar este artículo (APA):

García, M. \& Vélez, H. (2018). Exploración de las características psicométricas del inventario de estrés ocupacional - OSI para el contexto colombiano. Psicogente, 21(39), 140-161. http://doi.org/10.17081/psico.21.39.2828 


\section{INTRODUCCIÓN}

El estrés es un concepto dinámico en donde intervienen variables tanto del entorno como individuales, las cuales generan variedad de sensaciones y emociones relacionadas con presiones a las que se enfrenta un organismo (Dahl, 2010). La respuesta al estrés no necesariamente tiene un carácter negativo, puesto que puede ser necesaria para la supervivencia y actuar efectivamente para afrontarse a situaciones del diario vivir. Este aspecto es conocido como eustres o estrés bueno, el cual ejerce una función de protección en el organismo, el distress o estrés negativo se genera cuando hay una actividad o factor muy intenso que puede afectar física y psicológicamente a los individuos (Cirera, Aparecida, Rueda \& Ferraz, 2012; Schneiderman, Ironson \& Siegel, 2005; Magaya, Asner-Self \& Schreiber, 2005).

\subsection{Estrés de origen laboral}

En el contexto laboral, el estrés es una problemática común, afectando directa o indirectamente el desarrollo de las funciones del trabajador en la organización y transciende a otras áreas de desarrollo humano como lo son la esfera cognoscitiva, afectiva y social (Ramos, Moreno \& Ales, 2015).

Las causas del estrés tienen su origen en factores ambientales como individuales. Los factores ambientales son: a) intrínsecos al trabajo: condiciones de trabajo inadecuadas, b) el papel de la organización: exigencias acerca del comportamiento adecuado en el desempeño de las diferentes funciones, c) relaciones en el trabajo y el desarrollo de la carrera la falta de seguridad en el trabajo y la estructura del clima organizacional, sin embargo existen otros factores que pueden intervenir en la organización como la presión por la productividad,la relación abusiva entre los supervisores y en este caso cuanto mayor sea la demanda menor será el control del trabajador, y más probable será que se desencadene el estrés promoviendo pérdidas tanto en las organizaciones como en el individuo (Sánchez, 2011).

En cuanto a los factores emocionales y cognitivos que pueden desarrollar tics nerviosos, agotamiento, despersonalización, la distracción, la falta o exceso de apetito, la impulsividad y la agresividad, sudoración, taquicardia y alteraciones en la respiración, igualmente a nivel cognitivo se produce baja concentración y mal humor, en el sistema motor las consecuencias se ven reflejadas al hablar rápido, tartamudeo, entre otras (Castillo, Torres, Ahumada, Cárdenas \& Licona, 2014; Aguirre \& Quijano, 2015).

El individuo empieza a percibir y vivir en su ámbito laboral diferentes tipos de estrés, el primero, hace referencia al ámbito personal, cuando este tiene una experiencia subjetiva le generan dudas y preocupaciones, ya que cree que no cuenta con los recursos adecuados o necesarios para responder a las exigencias, produciéndole así ansiedad y temor; el segundo, hace referencia al ámbito laboral donde se ven relacionados los aspectos del trabajo que generan un desequilibrio y preocupación por la falta de apoyo que la organización no le brinda a la persona, por ejemplo, cuando no existe un liderazgo, la sobrecarga laboral es demasiada o el individuo no conoce el rol que debe desempeñar, por último está el estrés social que hace referencia a aquella percepción que la 


\section{Mónica García Rubiano, Helena Vélez Botero}

persona tiene en cuanto al entorno que la rodea y que a la vez es visto como preocupante o frustrante (Rodríguez \& De Rivas, 2011; Arce, 2012; Walter \& Arias, 2012).

A consecuencia de esto, en el individuo se van generando fuentes potenciales de estrés que se viven enel ámbito laboral y que por ende afectan su bienestar, todas aquellas actividades, condiciones ambientales, características del trabajo, los horarios que deben cumplir o las responsabilidades que debe asumir, el salario, los beneficios, las relaciones interpersonales que establezcan en su entorno laboral, las habilidades técnicas, la tecnología que la organización tenga, los clientes, el contexto social, entre otros son fuentes de estrés (Peiró \& Rodríguez, 2008). Por otro lado se encuentran los factores vinculados a la tarea, como aquellos que se relacionan con la antigüedad del rol, la sobrecarga laboral que debe tener el empleado y que a la vez ha sido impuesta por la organización, la jornada laboral, el trabajo en grupo, los conflictos que se presentan con la empresa o los grupos de trabajo, las preferencias o encubrimientos (Arce, 2012; Walter \& Arias, 2012).

Así mismo en el ámbito organizacional, se incluyen fuentes estresantes como las que están relacionadas con las condiciones de trabajo enfocado esto en el diseño del puesto o la distribución física del trabajo, las presiones que surgen debido al papel que desempeña en la organización, o las que son creadas por otros empleados hacia el individuo y que por tal generan tensión, también como fuente de estrés está la estructura de la organización, las normas que se manejan y la toma de decisiones que se planteen, el estilo de liderazgo también influye mucho ya que si no es apropiado y en beneficio del individuo genera gran tensión, temor y ansiedad (Peiró, 2009). Las malas relaciones con sus compañeros, la falta de confianza con los mismos o sus superiores serán también fuentes potenciales de estrés (Beh \& Loo, 2012). El Conflicto Hombre-trabajo también es fuente de estrés, ya que cuando el estrés interfiere en el rol de trabajo del individuo perjudica la capacidad que este debe tener para asumir y cumplir las obligaciones tanto del hogar como del trabajo (Peiró \& Rodríguez, 2008; Rodríguez \& De Rivas, 2011).

\subsection{Evaluación del estrés laboral}

Debido a la importancia que ha cobrado el modelo transaccional, y a partir de los desarrollos teóricos sobre estrés principalmente de Lazarus y Folkman (1986), ha crecido el interés por conocer más que el estresor en sí mismo, la valoración que el individuo realiza sobre el mismo. De este modo y desde una perspectiva transaccional, los instrumentos para evaluar este fenómeno deben explicar y predecir el grado de estrés ocupacional de un sujeto indagando las interacciones entre estresores percibidos y recursos de afrontamiento.

A la fecha, es común, el hallazgo de innumerables estudios aplicados, donde se hace uso de baterías, cuestionarios y tests, que evalúan distintos fenómenos psicológicos, por el contrario, la investigación científica, se ha limitado en cuanto al abordaje disciplinar y desarrollo teórico. Esta evidencia, aporta luces, sobre el uso indiscriminado y poco selectivo de los instrumentos para evaluación psicológica. Según lo descrito en Carretero Dios y Pérez (2007), solo el 55 \% de 696 
publicaciones científicas donde se hizo uso de tests proporcionaban evidencia sobre la validez de las puntuaciones generadas por los instrumentos. Esta declaración, sugiere que en gran parte de los estudios que hacen uso de test, no se tienen en cuenta aspectos como las limitantes psicosociales implícitas a la hora de estudiar un fenómeno y el desarrollo científico proveniente de validaciones, validez o confiabilidad, es uno de los menos utilizados, ya que la idea de implementar teorías es cada vez más reducida en las publicaciones científicas.

De acuerdo con lo estimado, en psicología, se ha estandarizado el uso de herramientas para el desarrollo investigativo y para la medición en diferentes contextos, los cuales se engloban en categorías como cuestionarioso checklists (Merín-Reig, Cano-Vindel \& Tobal, 1995). Los cuestionarios y escalas son instrumentos estandarizados, que proveen un marco de referencia otorgado por la investigación precedente, sin embargo, en la mayoría de los casos, este tipo de instrumentos se encuentran envueltos en teorías desactualizadas o en su defecto no son validados para el cambio contextual en su aplicación (Merín-Reig, Cano-Vindel \& Tobal, 1995). Al respecto, López y Campos (2002) sugieren que es conveniente, para el estudio y la comprensión de un fenómeno complejo como el del estrés laboral, realizar aproximaciones lo más abarcadoras posibles, y por ende, postula diferentes variables para comprender el estrés laboral como lo son el estrés percibido, la percepción de apoyo social familiar; la percepción de apoyo social de las amistades; el nivel de sintomatología y el nivel de estrés generado por la preocupación u ocurrencia de sucesos de vida.

En Colombia, el estudio del estrés laboral no ha sido ajeno a investigaciones. Para el 2008, el Ministerio de Protección Social, encargado en Colombia de regular asuntos concernientes en el ámbito del trabajo y la salud, y por ende competente para generar los estamentos legales para la medición del estrés ocupacional, considerado dentro del panorama de riesgos psicosociales estableció mediante la Resolución 2646 de 2008, la necesidad de crear una batería estandarizada para el contexto colombiano, que puede ser utilizada en los siguientes casos: a) identificación y evaluación periódica de los factores de riesgo psicosocial intra y extra laboral, con el fin de dar cumplimiento al Capítulo II de la Resolución 2646 de 2008; b) vigilancia epidemiológica de los factores de riesgo psicosocial; c) análisis psicosocial en procesos de determinación de origen de enfermedades presumiblemente derivadas del estrés; d) análisis psicosocial en procesos de reubicación laboral; e) diseño, implementación y evaluación de medidas de intervención de factores psicosociales; f) diseño e implementación de acciones de promoción, prevención y mejoramiento de las condiciones de salud de los trabajadores.

\subsection{Inventario de estrés ocupacional-OSI}

Leibovich de Figueroa y Schmidt (2004) aseguran que el inventario de estrés ocupacional (OSI) de Osipow y Spokane (1987, citados por Hicks, Sabanci \& Bahr, 2015) ha sido desarrollado para elaborar diferentes medidas genéricas de estresores ocupacionales que tengan aplicación en diferentes ambientes laborales, y a su vez, es útil para ser aplicado en ambientes ocupacionales variados uniendo diferentes fuentes de estrés potenciales percibidas por los individuos (Hermosa \& Perilla, 2015). 


\section{Mónica García Rubiano, Helena Vélez Botero}

Leibovich de Figueroa y Schmidt (2004) aseguran que el inventario de estrés ocupacional (OSI) de Osipow y Spokane (1987, citados por Hicks, Sabanci \& Bahr, 2015) ha sido desarrollado para elaborar diferentes medidas genéricas de estresores ocupacionales que tengan aplicación en diferentes ambientes laborales, y a su vez, es útil para ser aplicado en ambientes ocupacionales variados uniendo diferentes fuentes de estrés potenciales percibidas por los individuos (Hermosa \& Perilla, 2015).

Por su parte, la validez del inventario de estrés ocupacional (OSI) versión argentina se obtuvo por medio de estudios factoriales, estudios para evaluar los resultados de los tratamientos indicados para el manejo del estrés, estudios de la tensión, el estrés y los recursos para afrontarlo en grupos seleccionados y estudios de correlación entre escalas. Estos últimos, indudablemente han recibido gran parte de la atención pues arrojan datos exactos sobre el instrumento. De esta manera, seencontró una correlación negativa de -0,24 entre la subescala de tensión y la subescala de recursos de afrontamiento, es decir entre mayor tensión menores recursos de afrontamiento; así como entre la subescala de roles ocupacionales y la de recursos de afrontamiento se encontró una correlación negativa de -0,25 (Schmidt et al., 2003).

Los autores de la versión argentina del instrumento (OSI) también rescatan correlaciones negativas significativas entre la subescala de tensión vocacional y afrontamiento racional cognitivo, entre tensión psicológica y recursos personales y tensión interpersonal (Schmidt, et al., 2003). Ahora bien, en la muestra argentina también se realizaron análisis de confiabilidad y validez tras realizar la traducción de los ítems que componían cada subescala. En cuanto a la confiabilidad, se encontró un coeficiente alfa de 0,85 para el cuestionario de roles ocupacionales, de 0,78 para el cuestionario de tensión personal, y para el cuestionario de recursos de afrontamiento se encontró un alfa de 0,72, dando como resultado una confiabilidad de 0,62 a 0,84, siendo así, aceptable para las escalas individuales (Schmidt et al., 2003).

Con el propósito de establecer las propiedades psicométricas del OSI, en la población colombiana se tuvieron en cuenta dos fases: a) adaptación lingüística, estudio realizado por García-Neira, Piraquive y Salazar (2012), y b) pilotaje del instrumento. Para la adaptación lingüística, se tuvo como base el movimiento de aspectos cognitivos de la metodología de encuestas (CASM), el cual tiene sus fundamentos en la psicología cognitiva y otras ciencias sociales (Padilla, García \& Gómez, 2007). Este movimiento asume que las respuestas a las preguntas de cuestionarios dependen de cuatro procesos cognitivos, de los cuales se utilizaron dos para hacer laadaptación lingüística y cultural del cuestionario: juicio de expertos y entrevistas cognitivas.

Para el juicio de expertos lo primero que se hizo fue el ajuste de los ítems al español de Colombia, para posteriormente enviarlo a jueces expertos ( 5 de contenido y 3 metodológicos), esta evaluación permitió analizar dos componentes para poder identificar los ítems target; uno de ellos es la equivalencia conceptual o en otras palabras, cuando el término de la versión argentina requiere de un cambio por su uso infrecuente en el léxico de la población colombiana. El otro componente es la comprensión, el cual contempla el sentido de la pregunta en su redacción para el significado. 


\section{Mónica García Rubiano, Helena Vélez Botero}

Se utilizó el coeficiente $V$ de Aiken para cuantificar la validez de contenido de estos 8 jueces expertos frente a una redacción alternativa de los ítems de la versión argentina del cuestionario OSI. De acuerdo con Merino-Soto y Segovia (2009), la V de Aiken es un intervalo de confianza que se ha utilizado frecuentemente para evaluar cuantitativamente la opinión de expertos frente a un material. La magnitud va desde 0,00 hasta 1,00 , donde 1,00 corresponde a un alto nivel de acuerdo entre los jueces frente a los ítems que obtienen mayor puntuación en la relevancia o contenido de los componentes del material. El límite crítico con este intervalo de confianza depende simplemente del investigador, en este caso se utilizó un nivel conservador de 0,70 (Charter, 2003; Merino-Soto, 2016).

Posteriormente, una vez identificados los ítems target se procede a realizar las entrevistas cognitivas (30), las cuales fueron grabadas en formato de audio y se transcribieron para poder conocer la comprensión e interpretación de los ítems target por parte de 30 participantes que fueron trabajadores colombianos y que pertenecen a empresas del sector público y privado.

En total fueron 9 ítems target los que se obtuvieron teniendo en cuenta dos criterios. El primer criterio es el estándar mínimo de exigencia de 0,70 en la puntuación de los ítems para concluir con un nivel aceptable de validez de contenido frente a los 2 componentes de aná- lisis (comprensión y coherencia). Para este caso los ítems que presentaron valores por debajo del estándar mínimo $(0,70)$ con la V de Aiken fueron: $9(0,66), 10(0,62), 11(0,66), 12(0,64), 16(0,64), 17(0,66), 26(0.50), 32$ $(0,68)$ y $44(0,64)$.

Una vez identificados los ítems target se identifica el tipo de fallo que presentaban los ítems, en este caso fueron: a) error de percepción en la cognición, b) correspondencia de la palabra clave, c) expresión clave, d) ajuste de la pregunta a la dimensión, e) elaboración del ítem, f) asociado con el cambio del objeto, y g) uso de la palabra que depende del contexto. Después de identificado el fallo, se evalúa la técnica de indagación que se utilizará para la realización de la entrevista cognitiva que para el caso fueron: a) parafraseo (basado en la comprensión directa del ítem. El entrevistado tiene que repetir el ítem con sus propias palabras), b) prueba centrada en el significado (investigar cómo el entrevistado ha entendido una palabra o término del ítem clave para la comprensión de la pregunta), c) verbalización del pensamiento (conocer los pensamientos del entrevistado cuando responde a la pregunta), y d) clasificación de viñetas (conocer la respuesta que daría el entrevistado si fuera otra persona en un situación determinada, según los objetivos del ítem) (Padilla, García \& Gómez, 2007).

Después de realizado el anterior procedimiento se realizan las entrevistas cognitivas. Se entiende por entrevista cognitiva, una entrevista semi-estructurada en donde el entrevistador indaga sobre las dificultades que se le presentan al entrevistado frente a la compresiónde las preguntas, las tareas de recuperación de información, la elaboración de la respuesta, etc. Para este caso se concluye en las entrevistas cognitivas: a) ítem 9, áreas y actividades no se refieren a lo mismo, b) ítem 10, las expresiones "razonable" y "lo que corresponde" no son lo mismo, c) ítem 11, desarrollo y progreso no dan cuenta de la misma magnitud. El progreso es una calificación más significativa, d) ítem 12, el término "acomoda" no es adecuado para la comprensión esperada del ítem, e) ítem 16, se mantiene la versión original, f) ítem 17, las palabras "éxito" y “reconocimiento" no tienen el mismo significado. 
Se abre la pregunta, g) ítem 26, la connotación de las palabras "jefe" y "superior" pueden ser distintas, por ello es necesario evaluar la pertinencia de su uso, h) ítem 36, el uso de la palabra "acosado" no es adecuado para nuestro contexto, i) ítem 44, el uso de las palabras "buscar" y "reconocer" no es el mismo. Por tanto el documento quedó conformado por 141 ítems.

Finalmente para el pilotaje del cuestionario y poder establecer sus características psicométricas, se aplicó el instrumento a 500 trabajadores de diferentes sectores público y privado. Con la información recolectada se explorarán las características psicométricas del inventario de estrés ocupacional (OSI), el cual puede ser una herramienta fiable para ser utilizada tanto en el ámbito investigativo como en el ámbito laboral. Además del potencial evaluativo, una herramienta de medición válida permite plantear planes de intervención encaminados no solo a combatir el fenómeno, sino también a generar estrategias de prevención de la problemática desde una perspectiva del afrontamiento.

Este estudio tuvo como objetivo realizar una exploración de las características psicométricas del inventario de estrés ocupacional (OSI), para realizar los respectivos ajustes en su posterior validación para poblacióncolombiana. La importancia de realizar este tipo de estudios se encuentra en la estandarización de criterios válidos para cada región, en tanto es indispensable realizar estudios comparativos de carácter transcultural (Díaz \& Feldman, 2010).

\section{MÉTODO}

\subsection{Diseño}

Se utilizó un diseño transversal de tipo descriptivo-correlacional (Hernández, Fernández \& Baptista, 2006), en busca de identificar y describir las características psicométricas del inventario de estrés ocupacional (OSI) en su adaptación para población colombiana.

\subsection{Participantes}

Para la realización del pilotaje se contó con la participación voluntaria de 500 trabajadores vinculados a diferentes empresas y sectores. La muestra estuvo conformada en un $60,3 \%$ de hombres y un $39,7 \%$ de mujeres, entre los 17 y 71 años de edad, la vinculación laboral corresponde a un $28,7 \%$ empresas públicas, $67,7 \%$ privadas y 3,7 \% mixtas, la vinculación en su mayoría corresponde a contratos a término indefinido $68 \%$ y termino fijo el $32 \%$.

\subsection{Instrumentos}

Se empleó el inventario de estrés ocupacional (OSI, por sus iniciales en inglés) construido por Osipow y Spokane (1987, citados por Hicks, Sabanci \& Bahr, 2015) en la versión adaptada al español y a población argentina por Schmidt et al. (2003), adecuado lingüísticamente para población colombiana por García-Neira, Piraquive y Salazar (2012).

El instrumento cuenta con un total de 141 reactivos tipo Likert que indagan por la frecuencia de ocurrencia de determinados eventos, con opciones de respuesta que van desde " 1 " nunca, hasta 
"4" siempre. Está dividido en 3 escalas y 9 subescalas que exploran en detalle las dimensiones más destacadas en el fenómeno del estrés (Ver Tabla 1).

Tabla 1.

Escalas y subescalas del OSI

\begin{tabular}{clc}
\hline \multicolumn{1}{c}{ Escala } & \multicolumn{1}{c}{ SubEscala } & Número de ltems \\
\hline Roles Ocupacionales (ORQ) & Sobrecarga ocupacional (RO) & 10 \\
& Insuficiencia del rol (RI) & 11 \\
& Ambigüedad del rol (RA) & 10 \\
& Fronteras del rol (RB) & 10 \\
& Responsabilidad (R) & 10 \\
& Ambiente físico (PE) & 10 \\
Tensión Personal (PSQ) & Tensión vocacional (VS) & 10 \\
& Tensión psicológica (PSY) & 10 \\
& Tensión interpersonal (IS) & 10 \\
& Tensión física (PHS) & 10 \\
Recursos Personales (PRQ) & Recreación (RE) & 10 \\
& Autocuidado (SC) & 10 \\
& Soporte Social (SS) & 10 \\
& Afrontamiento racional/cognitivo (RC) & 10 \\
\hline
\end{tabular}

\subsection{Procedimiento}

Para aplicar el cuestionario se crearon dos versiones, una electrónica que se envió mediante correo masivo, se compartió en redes sociales y se difundió a través de medios electrónicos por canales de comunicación propios de empresas; la versión impresa se aplicó de manera masiva en el ámbito empresarial.

Una vez recolectada la información se consolidó una base de datos en Excel, en la cual se depuró lainformación imputando los datos faltantes (CarracedoMartínez \& Figueiras, 2006). Posteriormente se realizó el análisis estadístico de los datos con SPSS versión 22, comparando las características del instrumento con la versión original.

\subsection{Análisis de datos}

Se realizó con el paquete estadístico SPSS-22. Se analizaron las propiedades psicométricas relacionadas con la dificultad y discriminación de los ítems a través de la prueba t, la confiabilidad a través del coeficiente de Cronbach y la validez factorial mediante un análisis de máxima verosimilitud con rotación varimax.

\subsection{Consideraciones éticas}

Teniendo en cuenta los lineamientos de la Resolución No. 008430 de 1993 por la cual se establecen las normas científicas, técnicas y administrativas para la investigación en salud, la presente investigación tuvo en cuenta aspectos como los mencionados el artículo 6 del capítulo II, respecto al consentimiento informado, se contará con la autorización de la empresa para llevar a cabo la 
investigación, y con la aprobación del proyecto por parte de la institución investigadora que para el caso es la Universidad Católica de Colombia. Con respecto al anonimato mencionado en el artículo 8 del capítulo II, para la investigación, se protegerá la privacidad de los participantes, los cuales serán identificados solo cuando el estudio lo requiera.

Se ha considerado según el artículo 11 del capítulo II, que el presente estudio es una investigación sin riesgo debido a que no se realiza ninguna intervención o modificación intencionada de las variables biológicas, fisiológicas, sicológicas o sociales de los individuos que participaron en el estudio.

\section{RESULTADOS}

La muestra participante en el presente estudio estuvo conformada en un $29,4 \%$ por hombres y en un $70,6 \%$ por mujeres, con una edad promedio de 37 años. Los trabajadores vinculados al estudio pertenecen a empresas públicas $(37,8 \%)$, privadas $(58,6 \%)$ y mixtas $(3,6 \%)$, y están vinculados en su mayoría mediante contrato a término indefinido $(47,8 \%)$, seguido por el contrato a término fijo $(29,8 \%)$ y prestación de servicios $(17,45 \%)$.

La Tabla 2 evidencia una adecuada capacidad de los ítems en cuanto a su poder discriminativo, en la medida en para todos se observa una significancia estadística de $p \leq 0,05$ dentro de la prueba t.

Tabla 2.

\begin{tabular}{|c|c|c|c|c|}
\hline $\begin{array}{l}\text { Íte } \\
\mathrm{m}\end{array}$ & $\begin{array}{c}\text { Medi } \\
\text { a }\end{array}$ & $\begin{array}{c}\text { Desviació } \\
n \\
\text { estándar }\end{array}$ & $\mathrm{t}$ & $\begin{array}{c}\text { Significanc } \\
\text { ia }\end{array}$ \\
\hline 1 & 2,66 & 0,93 & 63,76 & 0,000 \\
\hline 2 & 2,70 & 0,91 & 66,69 & 0,000 \\
\hline 3 & 1,94 & 0,88 & 48,97 & 0,000 \\
\hline 4 & 2,12 & 1,03 & 45,77 & 0,000 \\
\hline 5 & 2,01 & 0,88 & 51,03 & 0,000 \\
\hline 6 & 1,42 & 0,61 & 52,22 & 0,000 \\
\hline 7 & 2,49 & 0,86 & 64,70 & 0,000 \\
\hline 8 & 2,36 & 0,89 & 59,45 & 0,000 \\
\hline 9 & 2,77 & 0,90 & 69,23 & 0,000 \\
\hline 10 & 2,18 & 0,95 & 51,10 & 0,000 \\
\hline 11 & 2,40 & 0,96 & 56,05 & 0,000 \\
\hline 12 & 2,02 & 0,90 & 50,36 & 0,000 \\
\hline 13 & 1,73 & 0,80 & 48,66 & 0,000 \\
\hline 14 & 1,97 & 0,85 & 51,99 & 0,000 \\
\hline 15 & 1,74 & 0,80 & 48,63 & 0,000 \\
\hline 16 & 2,06 & 1,00 & 46,29 & 0,000 \\
\hline 17 & 2,28 & 0,96 & 53,33 & 0,000 \\
\hline 18 & 2,40 & 0,95 & 56,42 & 0,000 \\
\hline 19 & 2,62 & 0,99 & 59,07 & 0,000 \\
\hline 20 & 1,89 & 0,88 & 47,94 & 0,000 \\
\hline 21 & 1,89 & 0,83 & 51,13 & 0,000 \\
\hline 22 & 2,72 & 0,99 & 61,31 & 0,000 \\
\hline 23 & 1,74 & 0,81 & 48,16 & 0,000 \\
\hline 24 & 1,71 & 0,85 & 44,87 & 0,000 \\
\hline 25 & 1,70 & 0,77 & 49,29 & 0,000 \\
\hline 26 & 1,83 & 0,74 & 55,17 & 0,000 \\
\hline 27 & 1,94 & 0,91 & 47,50 & 0,000 \\
\hline
\end{tabular}

\begin{tabular}{|c|c|c|c|c|}
\hline $\begin{array}{l}\text { Íte } \\
\mathrm{m}\end{array}$ & $\begin{array}{l}\text { Medi } \\
\text { a }\end{array}$ & $\begin{array}{c}\text { Desviació } \\
\text { n } \\
\text { estándar }\end{array}$ & $\mathrm{t}$ & $\begin{array}{c}\text { Significanc } \\
\text { ia }\end{array}$ \\
\hline 71 & 1,90 & 0,53 & 79,92 & 0,000 \\
\hline 72 & 1,80 & 0,79 & 50,90 & 0,000 \\
\hline 73 & 1,81 & 0,86 & 47,33 & 0,000 \\
\hline 74 & 1,84 & 0,82 & 50,26 & 0,000 \\
\hline 75 & 2,21 & 0,81 & 61,43 & 0,000 \\
\hline 76 & 1,83 & 0,79 & 51,43 & 0,000 \\
\hline 77 & 1,59 & 0,74 & 48,05 & 0,000 \\
\hline 78 & 1,58 & 0,73 & 48,38 & 0,000 \\
\hline 79 & 2,13 & 0,86 & 55,26 & 0,000 \\
\hline 80 & 1,93 & 0,78 & 55,15 & 0,000 \\
\hline 81 & 1,92 & 0,73 & 59,09 & 0,000 \\
\hline 82 & 2,55 & 0,95 & 60,17 & 0,000 \\
\hline 83 & 1,76 & 0,76 & 52,09 & 0,000 \\
\hline 84 & 1,39 & 0,60 & 51,70 & 0,000 \\
\hline 85 & 1,98 & 1,02 & 43,38 & 0,000 \\
\hline 86 & 2,18 & 0,89 & 55,01 & 0,000 \\
\hline 87 & 1,74 & 0,72 & 54,30 & 0,000 \\
\hline 88 & 1,81 & 0,77 & 52,18 & 0,000 \\
\hline 89 & 2,39 & 0,84 & 63,58 & 0,000 \\
\hline 90 & 2,74 & 0,94 & 65,08 & 0,000 \\
\hline 91 & 2,11 & 0,91 & 51,74 & 0,000 \\
\hline 92 & 2,01 & 1,02 & 44,25 & 0,000 \\
\hline 93 & 2,07 & 1,00 & 46,02 & 0,000 \\
\hline 94 & 1,15 & 0,48 & 53,26 & 0,000 \\
\hline 95 & 2,35 & 0,95 & 55,29 & 0,000 \\
\hline 96 & 2,26 & 0,91 & 55,32 & 0,000 \\
\hline 97 & 1,88 & 0,92 & 45,74 & 0,000 \\
\hline
\end{tabular}


Mónica García Rubiano, Helena Vélez Botero

\begin{tabular}{|c|c|c|c|c|c|c|c|c|c|}
\hline 28 & 1,26 & 0,54 & 52,05 & 0,000 & 98 & 1,88 & 0,92 & 45,54 & 0,000 \\
\hline 29 & 1,43 & 0,64 & 50,05 & 0,000 & 99 & 1,71 & 0,89 & 43,20 & 0,000 \\
\hline 30 & 1,89 & 0,90 & 46,89 & 0,000 & 100 & 2,07 & 0,95 & 48,93 & 0,000 \\
\hline 31 & 2,16 & 1,06 & 45,74 & 0,000 & 101 & 2,00 & 0,89 & 50,46 & 0,000 \\
\hline 32 & 1,79 & 0,82 & 48,56 & 0,000 & 102 & 2,37 & 1,08 & 48,99 & 0,000 \\
\hline 33 & 3,03 & 0,96 & 70,22 & 0,000 & 103 & 2,84 & 0,90 & 70,26 & 0,000 \\
\hline 34 & 2,05 & 0,98 & 47,05 & 0,000 & 104 & 2,75 & 0,88 & 69,59 & 0,000 \\
\hline 35 & 1,91 & 0,88 & 48,83 & 0,000 & 105 & 2,80 & 0,85 & 73,83 & 0,000 \\
\hline 36 & 1,72 & 0,78 & 49,73 & 0,000 & 106 & 2,06 & 0,83 & 55,59 & 0,000 \\
\hline 37 & 1,81 & 0,91 & 44,22 & 0,000 & 107 & 1,94 & 0,95 & 45,85 & 0,000 \\
\hline 38 & 1,44 & 0,73 & 43,89 & 0,000 & 108 & 1,52 & 0,76 & 44,87 & 0,000 \\
\hline 39 & 1,73 & 0,94 & 41,05 & 0,000 & 109 & 2,81 & 0,99 & 63,52 & 0,000 \\
\hline \multirow[t]{2}{*}{40} & 3,55 & 0,73 & 108,8 & 0,000 & 110 & 2,17 & 0,96 & 50,60 & 0,000 \\
\hline & & & 6 & & 111 & 2,76 & 0,98 & 63,11 & 0,000 \\
\hline 41 & 1,45 & 0,68 & 47,37 & 0,000 & 112 & 2,49 & 0,95 & 58,65 & 0,000 \\
\hline 42 & 2,34 & 1,02 & 51,30 & 0,000 & 113 & 2,29 & 0,98 & 52,16 & 0,000 \\
\hline 43 & 1,87 & 0,90 & 46,14 & 0,000 & 114 & 3,49 & 0,87 & 90,08 & 0,000 \\
\hline 44 & 2,37 & 1,20 & 43,98 & 0,000 & 115 & 2,08 & 1,03 & 45,17 & 0,000 \\
\hline 45 & 2,72 & 0,96 & 63,72 & 0,000 & 116 & 1,70 & 0,89 & 42,83 & 0,000 \\
\hline 46 & 2,46 & 1,07 & 51,55 & 0,000 & 117 & 2,57 & 0,94 & 61,30 & 0,000 \\
\hline 47 & 3,11 & 0,87 & 79,59 & 0,000 & 118 & 2,92 & 1,04 & 62,74 & 0,000 \\
\hline 48 & 1,99 & 0,77 & 57,57 & 0,000 & 119 & 1,79 & 0,93 & 42,87 & 0,000 \\
\hline 49 & 2,21 & 0,98 & 50,56 & 0,000 & 120 & 1,68 & 0,84 & 45,09 & 0,000 \\
\hline 50 & 2,53 & 0,84 & 67,50 & 0,000 & 121 & 2,47 & 0,89 & 62,23 & 0,000 \\
\hline 51 & 1,86 & 0,74 & 56,45 & 0,000 & 122 & 3,69 & 0,62 & 132,4 & 0,000 \\
\hline 52 & 2,10 & 1,02 & 46,13 & 0,000 & & & & 9 & \\
\hline 53 & 1,57 & 0,88 & 39,77 & 0,000 & 123 & 3,07 & 0,99 & 69,66 & 0,000 \\
\hline 54 & 1,85 & 0,99 & 42,08 & 0,000 & 124 & 3,16 & 0,84 & 83,75 & 0,000 \\
\hline 55 & 1,72 & 0,91 & 42,15 & 0,000 & 125 & 3,48 & 0,79 & 98,87 & 0,000 \\
\hline 56 & 1,56 & 0,85 & 41,29 & 0,000 & 126 & 3,45 & 0,82 & 94,25 & 0,000 \\
\hline 57 & 1,60 & 0,85 & 42,12 & 0,000 & 127 & 3,44 & 0,85 & 90,99 & 0,000 \\
\hline 58 & 2,13 & 0,98 & 48,64 & 0,000 & 128 & 3,58 & 0,67 & 120,2 & 0,000 \\
\hline 59 & 1,27 & 0,63 & 44,78 & 0,000 & & & & 9 & \\
\hline 60 & 1,45 & 0,78 & 41,56 & 0,000 & 129 & 3,62 & 0,71 & 114,2 & 0,000 \\
\hline 61 & 1,13 & 0,46 & 54,87 & 0,000 & & & & 9 & \\
\hline 62 & 1,83 & 1,02 & 39,93 & 0,000 & 130 & 3,22 & 0,90 & 80,25 & 0,000 \\
\hline 63 & 1,57 & 0,75 & 46,89 & 0,000 & 131 & 2,62 & 1,03 & 57,07 & 0,000 \\
\hline 64 & 1,68 & 0,77 & 48,54 & 0,000 & 132 & 2,64 & 0,98 & 60,18 & 0,000 \\
\hline 65 & 1,68 & 0,74 & 50,71 & 0,000 & 133 & 2,82 & 0,97 & 64,71 & 0,000 \\
\hline 66 & 1,10 & 0,39 & 63,05 & 0,000 & 134 & 2,65 & 0,86 & 68,65 & 0,000 \\
\hline 67 & 1,55 & 0,68 & 51,01 & 0,000 & 135 & 2,88 & 0,81 & 79,15 & 0,000 \\
\hline 68 & 1,31 & 0,57 & 50,93 & 0,000 & 136 & 2,97 & 0,82 & 80,94 & 0,000 \\
\hline 69 & 1,96 & 0,91 & 48,06 & 0,000 & 137 & 2,20 & 0,98 & 50,13 & 0,000 \\
\hline \multirow[t]{4}{*}{70} & 1,79 & 0,76 & 52,89 & 0,000 & 138 & 3,15 & 0,73 & 96,39 & 0,000 \\
\hline & & & & & 139 & 3,31 & 0,72 & $\begin{array}{r}102,3 \\
8\end{array}$ & 0,000 \\
\hline & & & & & 140 & 3,44 & 0,66 & $\begin{array}{r}116,0 \\
6\end{array}$ & 0,000 \\
\hline & & & & & 141 & 3,47 & 0,64 & 121,0 & 0,000 \\
\hline
\end{tabular}

De forma complementaria, el análisis de correlaciones evidencia asociaciones significativas entre las escalas y subescalas. Así pues, la escala "Roles Ocupacionales" muestra relaciones medias-altas significativas con las6 subescalas que la componen (RO, RI, RA, RB, R, PE), la escala "Tensión Personal" tiene relaciones fuertes y significativas con las 4 subescalas que la componen (VS, PSY, IS, PHS), así mismo la escala "Recursos Personales" tiene relaciones fuertes y significativas con las 4 
subescalas que la componen (RE, SC, SS, RC); mientras que con esta escala las demás subescalas muestran correlaciones negativas (ver Tabla 3).

Tabla 3.

Correlaciones entre escalas y subescalas

\begin{tabular}{|c|c|c|c|c|}
\hline \multirow[t]{2}{*}{ Escala } & \multirow[t]{2}{*}{ Sub Escala } & \multicolumn{3}{|c|}{ Escala } \\
\hline & & $\begin{array}{c}\text { Roles Ocupacionales } \\
\text { (ORQ) }\end{array}$ & $\begin{array}{c}\text { Tensión Personal } \\
\text { (PSQ) }\end{array}$ & $\begin{array}{c}\text { Recursos Personales } \\
\text { (PRQ) }\end{array}$ \\
\hline \multirow[t]{6}{*}{ ORQ } & Sobrecarga ocupacional (RO) &, $702^{* *}$ & $498^{* *}$ &,$- 386^{* *}$ \\
\hline & Insuficiencia del rol (RI) &, $679^{* *}$ &, $475^{* *}$ &,$- 374^{* *}$ \\
\hline & Ambigüedad del rol (RA) &, $728^{* *}$ &, $531^{* *}$ &,$- 430^{* *}$ \\
\hline & Fronteras del rol (RB) &, $794^{* *}$ &, $545^{* *}$ &,$- 382^{* *}$ \\
\hline & Responsabilidad (R) &, $540^{* *}$ &, $327^{* *}$ &,$- 186^{* *}$ \\
\hline & Ambiente físico (PE) &, $572^{* *}$ &, $302^{* *}$ &,$- 186^{* *}$ \\
\hline \multirow[t]{4}{*}{ PSQ } & Tensión vocacional (VS) &, $633^{* *}$ &, $778^{* *}$ &,$- 452^{* *}$ \\
\hline & Tensión psicológica (PSY) & $605^{* *}$ & $889^{* *}$ &,$- 532^{* *}$ \\
\hline & Tensión interpersonal (IS) &, $509^{* *}$ &, $833^{* *}$ &,$- 517^{* *}$ \\
\hline & Tensión física (PHS) &, $565^{* *}$ &, $908^{* *}$ &,$- 576^{* *}$ \\
\hline \multirow[t]{4}{*}{ PRQ } & Recreación (RE) &,$- 438^{* *}$ &,$- 530^{* *}$ &, $836^{* *}$ \\
\hline & Autocuidado (SC) &,$- 352^{* *}$ &,$- 503^{* *}$ &, $814^{* *}$ \\
\hline & Soporte Social (SS) &,$- 352^{* *}$ &,$- 422^{* *}$ &, $711^{* *}$ \\
\hline & Afrontamiento racional/cognitivo (RC) &,$- 372^{* *}$ &,$- 445^{* *}$ &, $742^{* *}$ \\
\hline
\end{tabular}

La Tabla 4 presenta el análisis de las relaciones entre las escalas y subescalas del instrumento con relación a los ítems que las conforman. Los resultados muestran que los ítems cada una de las escalas en su mayoría correlacionan significativamente con la escala, y los ítems de cada subescala se asocian significativamente con las subescalas a las que pertenecen.

Tabla 4.

Correlaciones entre ítems, subescalas y escalas

\begin{tabular}{|c|c|c|c|c|c|c|c|c|c|c|c|c|c|c|c|c|c|c|c|}
\hline \multirow{2}{*}{$\begin{array}{c}\text { Íte } \\
\text { m }\end{array}$} & \multicolumn{7}{|c|}{ Escala y subescalas } & \multirow{2}{*}{$\begin{array}{c}\text { Íte } \\
\mathrm{m}\end{array}$} & \multicolumn{5}{|c|}{ Escala y subescalas } & \multirow{2}{*}{$\begin{array}{l}\text { Íte } \\
\mathrm{m}\end{array}$} & \multicolumn{5}{|c|}{ Escala y subescalas } \\
\hline & $\begin{array}{c}\text { OR } \\
Q\end{array}$ & RO & RI & RA & RB & $\mathrm{R}$ & $\mathrm{PE}$ & & PSQ & VS & PSY & IS & PHS & & $\begin{array}{c}P R \\
Q\end{array}$ & $\mathrm{RE}$ & SC & SS & $\mathrm{RC}$ \\
\hline 1 & $\begin{array}{l}\text {,47 } \\
0^{* *}\end{array}$ & $\begin{array}{l}, 75 \\
4^{* *}\end{array}$ & $\begin{array}{r}10 \\
8^{*}\end{array}$ & $\begin{array}{l}, 21 \\
5^{* *}\end{array}$ & $\begin{array}{l}, 28 \\
0^{* *}\end{array}$ & $\begin{array}{l}38 \\
8^{* *}\end{array}$ & $\begin{array}{l}14 \\
6^{* *}\end{array}$ & 1 & $\begin{array}{l}, 22 \\
2^{* *}\end{array}$ & $\begin{array}{l}43 \\
7^{* *}\end{array}$ &, 12 & $\begin{array}{l}15 \\
1^{* *}\end{array}$ & $\begin{array}{l}, 13 \\
3^{* *}\end{array}$ & 1 & $\begin{array}{l}, 31 \\
7^{* *}\end{array}$ & 42 & ,19 & ,19 & $\begin{array}{l}16 \\
2^{* *}\end{array}$ \\
\hline 2 & $\begin{array}{l}\text {,39 } \\
0^{* *}\end{array}$ & $\begin{array}{l}, 71 \\
3^{* *}\end{array}$ & $\begin{array}{r}03 \\
1\end{array}$ & $\begin{array}{l}15 \\
7^{*}\end{array}$ & & $\begin{array}{l}\text {,35 } \\
9^{* *}\end{array}$ & $\begin{array}{l}10 \\
7^{*}\end{array}$ & 2 & $\begin{array}{l}, 66 \\
3^{* *}\end{array}$ & $\begin{array}{l}, 71 \\
2^{\text {** }}\end{array}$ & & & $\begin{array}{l}, 54 \\
0^{* *}\end{array}$ & 2 & $\begin{array}{l}, 61 \\
1^{* *}\end{array}$ & & 0 & 1 & $\begin{array}{l}42 \\
4^{* *}\end{array}$ \\
\hline 3 & $\begin{array}{l}46 \\
3^{* *}\end{array}$ & $\begin{array}{l}, 50 \\
9^{* *}\end{array}$ & $\begin{array}{l}24 \\
6^{* *}\end{array}$ & $\begin{array}{l}36 \\
2^{* *}\end{array}$ & & $\begin{array}{l}, 20 \\
0^{* *}\end{array}$ & $\begin{array}{l}19 \\
9^{* *}\end{array}$ & 3 & $\begin{array}{l}, 63 \\
9^{* *}\end{array}$ & $\begin{array}{l}, 71 \\
8^{* *}\end{array}$ & & & $\begin{array}{l}, 51 \\
4^{* *}\end{array}$ & 3 & & & & & $\begin{array}{l}39 \\
2^{* * *}\end{array}$ \\
\hline 4 & $\begin{array}{c}, 34 \\
1^{* *}\end{array}$ & $\begin{array}{l}, 58 \\
9^{* *}\end{array}$ & $\begin{array}{r}, 04 \\
0\end{array}$ & $\begin{array}{l}21 \\
1^{* *}\end{array}$ & $\begin{array}{l}, 18 \\
4^{* *}\end{array}$ & $\begin{array}{l}, 24 \\
9^{* *}\end{array}$ & $\begin{array}{r}10 \\
4^{*}\end{array}$ & 4 & $\begin{array}{l}, 53 \\
6^{* *}\end{array}$ & & & & $\begin{array}{l}, 42 \\
4^{* *}\end{array}$ & 4 & $\begin{array}{l}, 33 \\
6^{* *}\end{array}$ & $\begin{array}{l}, 44 \\
3^{* *}\end{array}$ & $\begin{array}{l}, 21 \\
5^{* *}\end{array}$ & $\begin{array}{l}, 22 \\
6^{* *}\end{array}$ & $\begin{array}{l}14 \\
7^{* *}\end{array}$ \\
\hline 5 & $\begin{array}{l}49 \\
1^{* *}\end{array}$ & $\begin{array}{l}44 \\
7^{* *}\end{array}$ & $\begin{array}{l}29 \\
7^{* *}\end{array}$ & $\begin{array}{l}35 \\
7^{* *}\end{array}$ & $\begin{array}{l}, 37 \\
8^{* *}\end{array}$ & $\begin{array}{l}16 \\
4^{* *}\end{array}$ & $\begin{array}{l}32 \\
3^{* *}\end{array}$ & 5 & $\begin{array}{l}, 22 \\
3^{* *}\end{array}$ & $\begin{array}{l}29 \\
7^{* *}\end{array}$ & $\begin{array}{l}15 \\
7^{* *}\end{array}$ & $\begin{array}{l}13 \\
2^{* *}\end{array}$ & $\begin{array}{l}, 20 \\
6^{* *}\end{array}$ & 5 & $\begin{array}{l}47 \\
8^{* *}\end{array}$ & $\begin{array}{l}62 \\
1^{* *}\end{array}$ & $\begin{array}{l}32 \\
9^{* *}\end{array}$ & $\begin{array}{l}29 \\
2^{* *}\end{array}$ & $\begin{array}{l}22 \\
8^{* *}\end{array}$ \\
\hline 6 & $\begin{array}{l}, 29 \\
5^{* *}\end{array}$ & $\begin{array}{l}, 20 \\
7^{* *}\end{array}$ & $\begin{array}{l}25 \\
0^{* *}\end{array}$ & $\begin{array}{l}40 \\
7^{* *}\end{array}$ & $\begin{array}{l}\text {,24 } \\
0^{* *}\end{array}$ & $\begin{array}{r}, 00 \\
5\end{array}$ & $\begin{array}{r}07 \\
0\end{array}$ & 6 & $\begin{array}{l}, 32 \\
6^{* *}\end{array}$ & $\begin{array}{l}48 \\
9^{* *}\end{array}$ & $\begin{array}{l}, 27 \\
6^{* *}\end{array}$ & $\begin{array}{l}19 \\
3^{* *}\end{array}$ & $\begin{array}{l}, 22 \\
8^{* *}\end{array}$ & 6 & $\begin{array}{l}, 53 \\
1^{* *}\end{array}$ & $\begin{array}{l}67 \\
4^{* *}\end{array}$ & & $\begin{array}{l}, 21 \\
6^{* *}\end{array}$ & $\begin{array}{l}24 \\
6^{* *}\end{array}$ \\
\hline 7 & $\begin{array}{l}, 46 \\
4^{* *}\end{array}$ & $\begin{array}{l}, 71 \\
6^{* *}\end{array}$ & $\begin{array}{l}13 \\
3^{* *}\end{array}$ & $\begin{array}{l}17 \\
9^{* *}\end{array}$ & $\begin{array}{l}, 25 \\
1^{* *}\end{array}$ & $\begin{array}{l}, 37 \\
0^{* *}\end{array}$ & $\begin{array}{l}20 \\
4^{* *}\end{array}$ & 7 & $\begin{array}{l}, 39 \\
9^{* *}\end{array}$ & $\begin{array}{l}46 \\
7^{* *}\end{array}$ & $\begin{array}{l}35 \\
0^{* *}\end{array}$ & $\begin{array}{l}, 29 \\
0^{* *}\end{array}$ & $\begin{array}{l}, 30 \\
5^{* *}\end{array}$ & 7 & $\begin{array}{l}, 29 \\
6^{* *}\end{array}$ & $\begin{array}{l}40 \\
0^{* *}\end{array}$ & $\begin{array}{l}, 22 \\
0^{* *}\end{array}$ & $\begin{array}{l}15 \\
2^{* *}\end{array}$ & $\begin{array}{l}13 \\
9^{* *}\end{array}$ \\
\hline 8 & $\begin{array}{l}\text {,47 } \\
1^{* *}\end{array}$ & $\begin{array}{l}, 70 \\
5^{* *}\end{array}$ & $\begin{array}{r}10 \\
9^{*}\end{array}$ & $\begin{array}{l}28 \\
0^{* *}\end{array}$ & $\begin{array}{l}, 26 \\
2^{* *}\end{array}$ & $\begin{array}{l}\text {,34 } \\
4^{* *}\end{array}$ & $\begin{array}{l}19 \\
3^{* *}\end{array}$ & 8 & $\begin{array}{l}, 44 \\
8^{* *}\end{array}$ & $\begin{array}{l}, 59 \\
7^{* *}\end{array}$ & $\begin{array}{l}41 \\
4^{* *}\end{array}$ & $\begin{array}{l}, 24 \\
3^{* *}\end{array}$ & $\begin{array}{l}34 \\
4^{* *}\end{array}$ & 8 & $\begin{array}{l}48 \\
9^{* *}\end{array}$ & $\begin{array}{l}, 54 \\
9^{* *}\end{array}$ & $\begin{array}{l}39 \\
0^{* *}\end{array}$ & $\begin{array}{l}, 27 \\
2^{* *}\end{array}$ & $\begin{array}{l}29 \\
8^{* *}\end{array}$ \\
\hline
\end{tabular}




\begin{tabular}{|c|c|c|c|c|c|c|c|c|c|c|c|c|c|c|c|c|c|c|c|}
\hline 9 & $\begin{array}{l}, 43 \\
0^{* *}\end{array}$ & $\begin{array}{l}, 72 \\
6^{* *}\end{array}$ & $\begin{array}{r}, 08 \\
2\end{array}$ & $\begin{array}{l}15 \\
9^{* *}\end{array}$ & $\begin{array}{l}, 22 \\
7^{* *}\end{array}$ & $\begin{array}{l}, 35 \\
6^{* *}\end{array}$ & $\begin{array}{l}17 \\
2^{* *}\end{array}$ & 9 & $\begin{array}{l}, 43 \\
4^{* *}\end{array}$ & $\begin{array}{l}, 59 \\
8^{* *}\end{array}$ & $\begin{array}{l}\text {,39 } \\
2^{* *}\end{array}$ & $\begin{array}{l}, 28 \\
4^{* *}\end{array}$ & $\begin{array}{l}29 \\
4^{* *}\end{array}$ & 9 & $\begin{array}{l}, 64 \\
4^{* *}\end{array}$ & $\begin{array}{l}, 71 \\
2^{* *}\end{array}$ & $\begin{array}{l}, 55 \\
6^{* *}\end{array}$ & $\begin{array}{l}, 34 \\
7^{* *}\end{array}$ & $\begin{array}{l}37 \\
3^{* *}\end{array}$ \\
\hline \multirow[t]{2}{*}{10} & ,56 & 77 & 22 & ,25 & 37, & ,36 & 24 & 10 & 20 & 27 & 14 & 19, & 14 & 10 & ,69 & ,77 &, 54 & ,37 & ,45 \\
\hline & $0^{* *}$ & $8^{* *}$ & $8^{* *}$ & $4^{* *}$ & $0^{* *}$ & $1^{* *}$ & $6^{* *}$ & & $9^{* *}$ & $0^{* *}$ & $2^{* *}$ & $6^{* *}$ & $7^{* *}$ & & $4^{* *}$ & $5^{* *}$ & $5^{* *}$ & $0^{* *}$ & $6^{* *}$ \\
\hline \multirow[t]{2}{*}{11} & ,50 & 15, & 77, & ,44 & ,43 & - & 13, & 11 & 67 & ,46 & ,72 & ,53 & ,56 & 11 & ,54 & ,47 & 63 & 23 & 31 \\
\hline & $5^{* *}$ & $0^{* *}$ & $3^{* *}$ & $0^{* *}$ & $7^{* *}$ & $\begin{array}{r}, 00 \\
8\end{array}$ & $5^{* *}$ & & $3^{* *}$ & $1^{* *}$ & $7^{* *}$ & $5^{* *}$ & $1^{* *}$ & & $0^{* *}$ & $4^{* *}$ & $9^{* *}$ & $4^{* *}$ & $9^{* *}$ \\
\hline \multirow[t]{2}{*}{12} & ,46 & 11 & ,74 & ,38 & ,42 & - & 13, & 12 & ,74 & ,51 & 81 & ,55 & ,62 & 12 & ,46 & ,37 & ,57 & 21 & 26 \\
\hline & $1^{* *}$ & $3^{*}$ & $7^{* *}$ & $7^{* *}$ & $7^{* *}$ & $\begin{array}{r}, 05 \\
6\end{array}$ & $7^{* *}$ & & $1^{* *}$ & $9^{* *}$ & $4^{* *}$ & $6^{* *}$ & $4^{* *}$ & & $3^{* *}$ & $0^{* *}$ & $6^{* *}$ & $4^{* *}$ & $7^{* *}$ \\
\hline \multirow[t]{2}{*}{13} & ,54 & 27 & 68 & 39, & 49 & 14 & 10, & 13 & 69, & ,51 & 79 & ,49 & ,55 & 13 & 25 & 10, & ,35 & 16, & 15, \\
\hline & $0^{* *}$ & $9^{* *}$ & $3^{* *}$ & $7^{* *}$ & $1^{* *}$ & $4^{* *}$ & $6^{*}$ & & $3^{* *}$ & $4^{* *}$ & $6^{* *}$ & $4^{* *}$ & $5^{* *}$ & & $5^{* *}$ & $9^{*}$ & $7^{* *}$ & $9^{* *}$ & $4^{* *}$ \\
\hline \multirow[t]{2}{*}{14} & ,39 & ,22 &, 50 & ,36 & ,34 & ,04 & 05 & 14 & ,60 & ,53 & ,68 & ,39 & ,46 & 14 & ,48 & ,47 & ,59 & 18, & 23 \\
\hline & $3^{* *}$ & $0^{* *}$ & $2^{* *}$ & $8^{* *}$ & $2^{* *}$ & 2 & 1 & & $3^{* *}$ & $8^{* *}$ & $6^{* *}$ & $7^{* *}$ & $0^{* *}$ & & $8^{* *}$ & $8^{* *}$ & $7^{* *}$ & $7^{* *}$ & $9^{* *}$ \\
\hline \multirow[t]{2}{*}{15} & ,48 & 14 & 69 & ,48 & ,47 & - & 10, & 15 & ,59, & ,34 & 61 & ,41 & ,59 & 15 & ,49 & ,35 & 68 & 17, & 30 \\
\hline & $4^{* *}$ & $2^{* *}$ & $8^{* *}$ & $2^{* *}$ & $6^{* *}$ & $\begin{array}{r}, 02 \\
0\end{array}$ & $1^{*}$ & & $7^{* *}$ & $9^{* *}$ & $8^{* *}$ & $4^{* *}$ & $9^{* *}$ & & $0^{* *}$ & $2^{* *}$ & $6^{* *}$ & $4^{* *}$ & $0^{* *}$ \\
\hline \multirow[t]{3}{*}{16} & ,55 & ,21 & ,83 & ,46 & ,51 & - & 09, & 16 & ,64 & ,45 & ,73 & ,46 &, 53 & 16 & ,55 & ,48 & ,59 & 29, & 34 \\
\hline & $0^{* *}$ & $6^{* *}$ & $2^{* *}$ & $3^{* *}$ & $3^{* *}$ & 01 & $6^{*}$ & & $1^{* *}$ & $1^{* *}$ & $1^{* *}$ & $0^{* *}$ & $1^{* *}$ & & $9^{* *}$ & $4^{* *}$ & $7^{* *}$ & $9^{* *}$ & $7^{* *}$ \\
\hline & & & & & & 3 & & & & & & & & & & & & & \\
\hline \multirow[t]{2}{*}{17} & ,59 & 23 & ,83 & ,48 & ,54 & ,04 & 14, & 17 & ,62 & ,47 & ,70 & ,44 & ,50 & 17 & ,39 & ,22 & ,53 & 25 & 20 \\
\hline & $4^{* *}$ & $7^{* *}$ & $7^{* *}$ & $4^{* *}$ & $8^{* *}$ & 7 & $0^{* *}$ & & $6^{* *}$ & $6^{* *}$ & $6^{* *}$ & $6^{* *}$ & $5^{* *}$ & & $2^{* *}$ & $3^{* *}$ & $3^{* *}$ & $4^{* *}$ & $0^{* *}$ \\
\hline \multirow[t]{2}{*}{18} & ,56 & 19, & 79, &, 50 &, 50 & 01 & 15 & 18 & ,70 & ,49 & ,77 & ,51 & ,60 & 18 & ,46 & ,30 & 64 & 14 & 34 \\
\hline & $0^{* *}$ & $7^{* *}$ & $0^{* *}$ & $0^{* *}$ & $2^{* *}$ & 3 & $7^{* *}$ & & $7^{* *}$ & $5^{* *}$ & $0^{* *}$ & $9^{* *}$ & $9^{* *}$ & & $7^{* *}$ & $9^{* *}$ & $7^{* *}$ & $7^{* *}$ & $4^{* *}$ \\
\hline \multirow[t]{2}{*}{19} & 14 & - & 31 & - & 13, & 11, & ,02 & 19 & 29, & ,30 & ,42 & 16 & 17, & 19 & ,46 & ,32 & ,65 & 15, & 30 \\
\hline & $2^{* *}$ & $\begin{array}{r}, 03 \\
6\end{array}$ & $2^{* *}$ & $\begin{array}{r}, 04 \\
0\end{array}$ & $8^{* *}$ & $9^{* *}$ & 8 & & $9^{* *}$ & $1^{* *}$ & $0^{* *}$ & $0^{* *}$ & $0^{* *}$ & & $4^{* *}$ & $\Lambda^{* *}$ & $5^{* *}$ & $3^{* *}$ & $2^{* *}$ \\
\hline \multirow[t]{2}{*}{20} & ,40 & ,02 & 69 & ,36 & ,35 & - & 12 & 20 & ,53 & ,52 & 61 & ,33 & ,37 & 20 & ,68 & ,65 & 61 & ,38 & 46 \\
\hline & $4^{* *}$ & 5 & $9^{* *}$ & $8^{* *}$ & $0^{* *}$ & $\begin{array}{r}, 03 \\
8\end{array}$ & $2^{* *}$ & & $3^{* *}$ & $5^{* *}$ & $9^{* *}$ & $9^{* *}$ & $7^{* *}$ & & $3^{* *}$ & $4^{* *}$ & $1^{* *}$ & $7^{* *}$ & $2^{* *}$ \\
\hline \multirow[t]{2}{*}{21} & ,36 & 19, & 44 & 21 & ,33 & 08, & 12 & 21 & 41 & 1,24 & ,24 &, 5 & ,37 & 21 & , 3 & 1 & 20, & , & 21 \\
\hline & $1^{* *}$ & $9^{* *}$ & $5^{* *}$ & $7^{* *}$ & $2^{* *}$ & $8^{*}$ & $1^{* *}$ & & $2^{* *}$ & $9^{* *}$ & $9^{* *}$ & $7^{* *}$ & $0^{* *}$ & & $2^{* *}$ & $3^{* *}$ & $3^{* *}$ & $3^{* *}$ & $9^{* *}$ \\
\hline \multirow[t]{2}{*}{22} & ,43 & 15, & 45 & 61 & ,35 & ,04 & 11 & 22 & ,47 & ,26 & ,35 & ,56 & ,42 & 22 & ,41 & ,27 & 24 & ,55 & 21 \\
\hline & $9^{* *}$ & $3^{* *}$ & $6^{* *}$ & $1^{* *}$ & $9^{* *}$ & 7 & $9^{* *}$ & & $6^{* *}$ & $9^{* *}$ & $2^{* *}$ & $9^{* *}$ & $3^{* *}$ & & $6^{* *}$ & $4^{* *}$ & $4^{* *}$ & $9^{* *}$ & $0^{* *}$ \\
\hline 23 & ,45 & 27 & ,43 & 67 & ,38 & - & ,04 & 23 & ,42 & 31 & ,35 & ,48 & ,33 & 23 & ,56 & 39 & 31 & 71 & 33 \\
\hline & $5^{* *}$ & $9^{* *}$ & $6^{* *}$ & $3^{* *}$ & $9^{* *}$ & $\begin{array}{r}01 \\
1\end{array}$ & 8 & & $9^{* *}$ & $7^{* *}$ & $4^{* *}$ & $3^{* *}$ & $1^{* *}$ & & $7^{* *}$ & $5^{* *}$ & $6^{* *}$ & $4^{* *}$ & $2^{* *}$ \\
\hline 24 & ,42 & 29 & 23 & ,49 & , 3 & 13, & 23 & 24 & ,33 & 1 & 2 & ,4 & ,24 & 24 & ,5 & 12 & 12 & p & 24 \\
\hline & $8^{* *}$ & $6^{* *}$ & $6^{* *}$ & $7^{* *}$ & $8^{* *}$ & $6^{* *}$ & $6^{* *}$ & & $0^{* *}$ & $1^{* *}$ & & $1^{* *}$ & $6^{* *}$ & & $3^{* *}$ & $0^{* *}$ & $2^{* *}$ & $0^{* *}$ & $7^{* *}$ \\
\hline 25 & ,32 & ,22 & 18, & ,53 & 25 & ,03 & 09, & 25 & ,40 & 25 & 29 & ,50 & ,32 & 25 & ,46 & ,26 & 17, & ,76 & 24 \\
\hline & $3^{* *}$ & $8^{* *}$ & $1^{* *}$ & $0^{* *}$ & $6^{* *}$ & 3 & $1^{*}$ & & $0^{* *}$ & $4^{* *}$ & $2^{* *}$ & $3^{* *}$ & $6^{* *}$ & & $3^{* *}$ & $1^{* *}$ & $2^{* *}$ & $1^{* *}$ & $3^{* *}$ \\
\hline 26 & ,37 & ,24 & ,23 & ,58 & ,26 & ,05 & 12 & 26 & ,51 & ,32 & 39, & ,63 & ,40 & 26 & ,47 & 28 & ,24 & ,71 & 21 \\
\hline & $3^{* *}$ & $7^{* *}$ & $8^{* *}$ & $3^{* *}$ & $7^{* *}$ & 7 & $0^{* *}$ & & $2^{* *}$ & $5^{* *}$ & $4^{* *}$ & $4^{* *}$ & $8^{* *}$ & & $2^{* *}$ & $7^{* *}$ & $3^{* *}$ & $2^{* *}$ & $7^{* *}$ \\
\hline 27 & ,52 & ,33 & 31 & ,53 & ,46 & 20 & 28 & 27 & ,49 & 39 & ,49 & ,47 & ,36 & 27 & ,49 & 29 & 25 & ,73 & 25 \\
\hline & $4^{* *}$ & $8^{* *}$ & $3^{* *}$ & $2^{* *}$ & $4^{* *}$ & $3^{* *}$ & $6^{* *}$ & & $6^{* *}$ & $2^{* *}$ & $4^{* *}$ & $0^{* *}$ & $2^{* *}$ & & $9^{* *}$ & $4^{* *}$ & $8^{* *}$ & $8^{* *}$ & $4^{* *}$ \\
\hline 28 & 27 & 12 & 15, & ,39 & 24 & ,05 & 14, & 28 & ,52 & 29, & ,35 & ,68 & ,46 & 28 & ,46 & ,24 & 21 & ,74 & 23, \\
\hline & $0^{* *}$ & $2^{* *}$ & $0^{* *}$ & $3^{* *}$ & $5^{* *}$ & 6 & $9^{* *}$ & & $8^{* *}$ & $7^{* *}$ & $7^{* *}$ & $5^{* *}$ & $1^{* *}$ & & $1^{* *}$ & $5^{* *}$ & $1^{* *}$ & $3^{* *}$ & $1^{* *}$ \\
\hline 29 & ,47 & 28 & ,32 & 67 & ,40 & 14 & 11 & 29 & ,56 & ,31 & ,37 & 69, &, 52 & 29 & ,51 & ,36 & 29, & ,66 & 24 \\
\hline & $9^{* *}$ & $6^{* *}$ & $3^{* *}$ & $4^{* *}$ & $0^{* *}$ & $9^{* *}$ & $6^{* *}$ & & $6^{* *}$ & $5^{* *}$ & $8^{* *}$ & $7^{* *}$ & $6^{* *}$ & & $1^{* *}$ & $9^{* *}$ & $8^{* *}$ & $5^{* *}$ & $8^{* *}$ \\
\hline 30 & ,54 & 28 & ,38 & ,72 & ,50 & 17, & 13, & 30 & ,66 & ,40 & ,51 & ,74 & ,58 & 30 & ,42 & ,36 & 29, & 41 & 23 \\
\hline & $2^{* *}$ & $0^{* *}$ & $9^{* *}$ & $3^{* *}$ & $8^{* *}$ & $5^{* *}$ & $9^{* *}$ & & $0^{* *}$ & $1^{* *}$ & $2^{* *}$ & $5^{* *}$ & $4^{* *}$ & & $3^{* *}$ & $3^{* *}$ & $2^{* *}$ & $7^{* *}$ & $5^{* *}$ \\
\hline 31 & 41 & 14 & 41 & ,64 & ,36 & ,00 & 07, & 31 & ,63 & 39 & ,48 & ,51 & ,72 & 31 &, 50 & ,42 & ,32 & ,32 & ,50 \\
\hline & $2^{* *}$ & $7^{* *}$ & $7^{* *}$ & $2^{* *}$ & $3^{* *}$ & 6 & 4 & & $7^{* *}$ & $1^{* *}$ & $1^{* *}$ & $9^{* *}$ & $1^{* *}$ & & $5^{* *}$ & $1^{* *}$ & $8^{* *}$ & $2^{* *}$ & $8^{* *}$ \\
\hline 32 & ,53 & ,33 & ,34 & ,46 & ,58 & ,25 & 23 & 32 & ,58 & ,31 & ,45 & ,44 & 68 & 32 & ,23 & ,08 & 13, & ,08 & 46 \\
\hline & $6^{* *}$ & $2^{* *}$ & $0^{* *}$ & $5^{* *}$ & $6^{* *}$ & $0^{* *}$ & $3^{* *}$ & & $0^{* *}$ & $2^{* *}$ & $6^{* *}$ & $8^{* *}$ & $3^{* *}$ & & $9^{* *}$ & 2 & $0^{* *}$ & $9^{*}$ & $2^{* *}$ \\
\hline 33 & ,25 & 28 & ,02 & - & 33, & 30, & 13, & 33 & 27, & 15, & 18 & ,24 & ,32 & 33 & ,50 & ,35 & ,36 & 22 & 63 \\
\hline & $4^{* *}$ & $4^{* *}$ & 2 & ,00 & $9^{* *}$ & $8^{* *}$ & $2^{* *}$ & & $9^{* *}$ & $6^{* *}$ & $7^{* *}$ & $8^{* *}$ & $8^{* *}$ & & $0^{* *}$ & $4^{* *}$ & $3^{* *}$ & $5^{* *}$ & $0^{* *}$ \\
\hline
\end{tabular}




\begin{tabular}{|c|c|c|c|c|c|c|c|c|c|c|c|c|c|c|c|c|c|c|c|}
\hline 34 & $\begin{array}{l}, 47 \\
4^{* *}\end{array}$ & $\begin{array}{l}35 \\
7^{* *}\end{array}$ & $\begin{array}{l}, 20 \\
6^{* *}\end{array}$ & $\begin{array}{l}, 27 \\
0^{* *}\end{array}$ & $\begin{array}{l}, 60 \\
3^{* *}\end{array}$ & $\begin{array}{l}30 \\
3^{* *}\end{array}$ & $\begin{array}{l}25 \\
0^{* *}\end{array}$ & 34 & $\begin{array}{l}, 75 \\
5^{* *}\end{array}$ & $\begin{array}{l}, 52 \\
3^{* *}\end{array}$ & $\begin{array}{l}, 59 \\
6^{* *}\end{array}$ & $\begin{array}{l}, 60 \\
0^{* *}\end{array}$ & $\begin{array}{l}80 \\
7^{* *}\end{array}$ & 34 & $\begin{array}{l}, 59 \\
4^{* *}\end{array}$ & $\begin{array}{l}44 \\
4^{* *}\end{array}$ & $\begin{array}{l}39 \\
3^{* *}\end{array}$ & $\begin{array}{l}, 28 \\
8^{* *}\end{array}$ & $\begin{array}{l}, 74 \\
3^{* *}\end{array}$ \\
\hline 35 & $\begin{array}{r}, 08 \\
4\end{array}$ & $\begin{array}{r}- \\
11 \\
0^{*}\end{array}$ & $\begin{array}{l}, 23 \\
8^{* *}\end{array}$ & $\begin{array}{l}, 27 \\
0^{* *}\end{array}$ & $\begin{array}{l}, 26 \\
4^{* *}\end{array}$ & $\begin{array}{r}- \\
, 29 \\
1^{* *}\end{array}$ & $\begin{array}{r}- \\
, 01 \\
9\end{array}$ & 35 & $\begin{array}{l}, 80 \\
7^{* *}\end{array}$ & $\begin{array}{l}57 \\
3^{* *}\end{array}$ & $\begin{array}{l}, 69 \\
4^{* *}\end{array}$ & $\begin{array}{l}, 64 \\
1^{* *}\end{array}$ & $\begin{array}{l}, 80 \\
8^{* *}\end{array}$ & 35 & $\begin{array}{l}, 59 \\
6^{* *}\end{array}$ & $\begin{array}{l}43 \\
7^{* *}\end{array}$ & $\begin{array}{l}41 \\
8^{* *}\end{array}$ & $\begin{array}{l}, 24 \\
9^{* *}\end{array}$ & $\begin{array}{l}, 77 \\
3^{* *}\end{array}$ \\
\hline 36 & $\begin{array}{l}, 60 \\
2^{* *}\end{array}$ & $\begin{array}{l}28 \\
9^{* *}\end{array}$ & $\begin{array}{l}, 71 \\
7^{* *}\end{array}$ & $\begin{array}{l}, 54 \\
3^{* *}\end{array}$ & $\begin{array}{l}63 \\
8^{* *}\end{array}$ & $\begin{array}{r}08 \\
2\end{array}$ & $\begin{array}{l}11 \\
5^{* *}\end{array}$ & 36 & $\begin{array}{l}, 69 \\
5^{* *}\end{array}$ & $\begin{array}{l}45 \\
4^{* *}\end{array}$ & $\begin{array}{l}, 61 \\
5^{* *}\end{array}$ & $\begin{array}{l}47 \\
3^{* *}\end{array}$ & $\begin{array}{l}, 76 \\
3^{* *}\end{array}$ & 36 & $\begin{array}{l}45 \\
7^{* *}\end{array}$ & $\begin{array}{l}30 \\
5^{* *}\end{array}$ & $\begin{array}{l}44 \\
0^{* *}\end{array}$ & $\begin{array}{l}15 \\
2^{* *}\end{array}$ & $\begin{array}{l}, 53 \\
7^{* *}\end{array}$ \\
\hline 37 & $\begin{array}{l}, 47 \\
9^{* *}\end{array}$ & $\begin{array}{l}, 26 \\
2^{* *}\end{array}$ & $\begin{array}{l}, 26 \\
6^{* *}\end{array}$ & $\begin{array}{l}, 36 \\
6^{* *}\end{array}$ & $\begin{array}{l}, 57 \\
2^{* *}\end{array}$ & $\begin{array}{l}21 \\
7^{* *}\end{array}$ & $\begin{array}{l}30 \\
9^{* *}\end{array}$ & 37 & $\begin{array}{l}, 70 \\
3^{* *}\end{array}$ & $\begin{array}{l}45 \\
2^{* *}\end{array}$ & $\begin{array}{l}, 56 \\
9^{* *}\end{array}$ & $\begin{array}{l}, 54 \\
7^{* *}\end{array}$ & $\begin{array}{l}76 \\
8^{* *}\end{array}$ & 37 & $\begin{array}{l}, 46 \\
5^{* *}\end{array}$ & $\begin{array}{l}23 \\
9^{* *}\end{array}$ & $\begin{array}{l}, 30 \\
7^{* *}\end{array}$ & $\begin{array}{l}, 26 \\
4^{* *}\end{array}$ & $\begin{array}{l}, 66 \\
0^{* *}\end{array}$ \\
\hline 38 & $\begin{array}{l}, 47 \\
2^{* *}\end{array}$ & $\begin{array}{l}19 \\
7^{* *}\end{array}$ & $\begin{array}{l}, 57 \\
4^{* *}\end{array}$ & $\begin{array}{l}43 \\
2^{* *}\end{array}$ & $\begin{array}{l}, 57 \\
3^{* *}\end{array}$ & $\begin{array}{r}, 00 \\
5\end{array}$ & $\begin{array}{r}10 \\
0^{*}\end{array}$ & 38 & $\begin{array}{l}, 59 \\
9^{* *}\end{array}$ & $\begin{array}{l}37 \\
4^{* *}\end{array}$ & $\begin{array}{l}44 \\
4^{* *}\end{array}$ & $\begin{array}{l}45 \\
8^{* *}\end{array}$ & $\begin{array}{l}, 70 \\
1^{* *}\end{array}$ & 38 & $\begin{array}{l}40 \\
8^{* *}\end{array}$ & $\begin{array}{l}, 20 \\
4^{* *}\end{array}$ & $\begin{array}{l}, 21 \\
3^{* *}\end{array}$ & $\begin{array}{l}, 21 \\
5^{* *}\end{array}$ & $\begin{array}{l}, 66 \\
5^{* *}\end{array}$ \\
\hline 39 & $\begin{array}{l}, 44 \\
9^{* *}\end{array}$ & $\begin{array}{l}, 21 \\
3^{* *}\end{array}$ & $\begin{array}{l}30 \\
9^{* *}\end{array}$ & $\begin{array}{l}, 39 \\
6^{* *}\end{array}$ & $\begin{array}{l}, 55 \\
0^{* *}\end{array}$ & $\begin{array}{l}18 \\
2^{* *}\end{array}$ & $\begin{array}{l}, 21 \\
2^{* *}\end{array}$ & 39 & $\begin{array}{l}75 \\
1^{* *}\end{array}$ & $\begin{array}{l}, 51 \\
4^{* *}\end{array}$ & $\begin{array}{l}, 58 \\
9^{* *}\end{array}$ & $\begin{array}{l}, 57 \\
8^{* *}\end{array}$ & $\begin{array}{l}, 82 \\
4^{* *}\end{array}$ & 39 & $\begin{array}{l}40 \\
6^{* *}\end{array}$ & $\begin{array}{l}19 \\
1^{* *}\end{array}$ & $\begin{array}{l}19 \\
6^{* *}\end{array}$ & $\begin{array}{l}, 26 \\
0^{* *}\end{array}$ & $\begin{array}{l}, 64 \\
2^{* *}\end{array}$ \\
\hline 40 & $\begin{array}{c}- \\
, 28 \\
0^{* *}\end{array}$ & $\begin{array}{r}- \\
13 \\
6^{* *}\end{array}$ & $\begin{array}{r}- \\
, 28 \\
5^{* *}\end{array}$ & $\begin{array}{r}- \\
, 38 \\
3^{* *}\end{array}$ & $\begin{array}{r}- \\
20 \\
2^{* *}\end{array}$ & $\begin{array}{r}- \\
, 01 \\
8\end{array}$ & $\begin{array}{r}- \\
07 \\
9\end{array}$ & 40 & $\begin{array}{l}, 71 \\
5^{* *}\end{array}$ & $\begin{array}{l}, 48 \\
6^{* *}\end{array}$ & $\begin{array}{l}, 57 \\
3^{* *}\end{array}$ & $\begin{array}{l}, 54 \\
4^{* *}\end{array}$ & $\begin{array}{l}, 78 \\
1^{* *}\end{array}$ & 40 & $\begin{array}{l}, 40 \\
7^{* *}\end{array}$ & $\begin{array}{l}, 22 \\
2^{* *}\end{array}$ & $\begin{array}{l}, 20 \\
4^{* *}\end{array}$ & $\begin{array}{l}, 24 \\
1^{* *}\end{array}$ & $\begin{array}{l}, 62 \\
2^{* *}\end{array}$ \\
\hline 41 & $\begin{array}{l}, 55 \\
4^{* *}\end{array}$ & $\begin{array}{l}, 26 \\
2^{* *}\end{array}$ & $\begin{array}{l}, 56 \\
0^{* *}\end{array}$ & $\begin{array}{l}, 49 \\
8^{* *}\end{array}$ & $\begin{array}{l}, 63 \\
1^{* *}\end{array}$ & $\begin{array}{r}, 09 \\
8^{*}\end{array}$ & $\begin{array}{l}18 \\
1^{* *}\end{array}$ & & & & & & & & & & & & \\
\hline 42 & $\begin{array}{l}, 25 \\
4^{* *}\end{array}$ & $\begin{array}{l}, 26 \\
5^{* *}\end{array}$ & $\begin{array}{r}, 00 \\
2\end{array}$ & $\begin{array}{r}, 00 \\
0\end{array}$ & $\begin{array}{r}, 05 \\
7\end{array}$ & $\begin{array}{l}, 50 \\
1^{* *}\end{array}$ & $\begin{array}{l}19 \\
9^{* *}\end{array}$ & & & & & & & & & & & & \\
\hline 43 & $\begin{array}{l}, 42 \\
2^{* *}\end{array}$ & $\begin{array}{l}37 \\
7^{* *}\end{array}$ & $\begin{array}{r}, 08 \\
1\end{array}$ & $\begin{array}{l}, 26 \\
2^{* *}\end{array}$ & $\begin{array}{l}28 \\
1^{* *}\end{array}$ & $\begin{array}{l}49 \\
9^{* *}\end{array}$ & $\begin{array}{l}23 \\
9^{* *}\end{array}$ & & & & & & & & & & & & \\
\hline 44 & $\begin{array}{l}11 \\
6^{* *}\end{array}$ & $\begin{array}{r}10 \\
8^{*}\end{array}$ & $\begin{array}{r}- \\
16 \\
7^{* *}\end{array}$ & $\begin{array}{r}- \\
, 09 \\
5^{*}\end{array}$ & $\begin{array}{r}- \\
, 05 \\
2\end{array}$ & $\begin{array}{l}61 \\
1^{* *}\end{array}$ & $\begin{array}{r}10 \\
0^{*}\end{array}$ & & & & & & & & & & & & \\
\hline 45 & $\begin{array}{l}15 \\
9^{* *}\end{array}$ & $\begin{array}{l}23 \\
3^{* *}\end{array}$ & $\begin{array}{r}- \\
, 15 \\
6^{* *}\end{array}$ & $\begin{array}{r}- \\
, 09 \\
4^{*}\end{array}$ & $\begin{array}{r}02 \\
0\end{array}$ & $\begin{array}{l}61 \\
0^{* *}\end{array}$ & $\begin{array}{r}07 \\
1\end{array}$ & & & & & & & & & & & & \\
\hline 46 & $\begin{array}{l}, 28 \\
6^{* *}\end{array}$ & $\begin{array}{l}, 30 \\
9^{* *}\end{array}$ & $\begin{array}{r}- \\
, 06 \\
3\end{array}$ & $\begin{array}{r}, 05 \\
8\end{array}$ & $\begin{array}{r}10 \\
5^{*}\end{array}$ & $\begin{array}{l}, 67 \\
5^{* *}\end{array}$ & $\begin{array}{r}10 \\
6^{*}\end{array}$ & & & & & & & & & & & & \\
\hline 47 & $\begin{array}{c}10 \\
9^{*}\end{array}$ & $\begin{array}{r}, 08 \\
7\end{array}$ & $\begin{array}{r}- \\
, 07 \\
8\end{array}$ & $\begin{array}{r}- \\
, 09 \\
8^{*}\end{array}$ & $\begin{array}{r}- \\
, 03 \\
9\end{array}$ & $\begin{array}{l}, 51 \\
7^{* *}\end{array}$ & $\begin{array}{r}, 06 \\
3\end{array}$ & & & & & & & & & & & & \\
\hline 48 & $\begin{array}{l}, 47 \\
0^{* *}\end{array}$ & $\begin{array}{l}32 \\
0^{* *}\end{array}$ & $\begin{array}{l}, 22 \\
4^{* *}\end{array}$ & $\begin{array}{l}, 29 \\
7^{* *}\end{array}$ & $\begin{array}{l}32 \\
1^{* *}\end{array}$ & $\begin{array}{l}47 \\
1^{* *}\end{array}$ & $\begin{array}{l}, 27 \\
2^{* *}\end{array}$ & & & & & & & & & & & & \\
\hline 49 & $\begin{array}{l}, 30 \\
2^{* *}\end{array}$ & $\begin{array}{l}, 22 \\
2^{* *}\end{array}$ & $\begin{array}{r}, 03 \\
6\end{array}$ & $\begin{array}{r}, 07 \\
4\end{array}$ & $\begin{array}{l}19 \\
0^{* *}\end{array}$ & $\begin{array}{l}, 51 \\
1^{* *}\end{array}$ & $\begin{array}{l}, 21 \\
3^{* *}\end{array}$ & & & & & & & & & & & & \\
\hline 50 & $\begin{array}{l}, 43 \\
6^{* *}\end{array}$ & $\begin{array}{l}27 \\
8^{* *}\end{array}$ & $\begin{array}{l}19 \\
8^{* *}\end{array}$ & $\begin{array}{l}17 \\
2^{* *}\end{array}$ & $\begin{array}{l}31 \\
1^{* *}\end{array}$ & $\begin{array}{l}, 53 \\
6^{* *}\end{array}$ & $\begin{array}{l}27 \\
7^{* *}\end{array}$ & & & & & & & & & & & & \\
\hline 51 & $\begin{array}{l}45 \\
7^{* *}\end{array}$ & $\begin{array}{l}, 30 \\
8^{* *}\end{array}$ & $\begin{array}{l}, 33 \\
5^{* *}\end{array}$ & $\begin{array}{l}, 34 \\
8^{* *}\end{array}$ & $\begin{array}{l}, 39 \\
5^{* *}\end{array}$ & $\begin{array}{l}, 30 \\
5^{* *}\end{array}$ & $\begin{array}{l}15 \\
0^{* *}\end{array}$ & & & & & & & & & & & & \\
\hline 52 & $\begin{array}{l}, 44 \\
8^{* *}\end{array}$ & $\begin{array}{l}29 \\
0^{* *}\end{array}$ & $\begin{array}{l}12 \\
8^{* *}\end{array}$ & $\begin{array}{l}14 \\
7^{* *}\end{array}$ & $\begin{array}{l}, 27 \\
8^{* *}\end{array}$ & $\begin{array}{l}25 \\
8^{* *}\end{array}$ & $\begin{array}{l}, 71 \\
0^{* *}\end{array}$ & & & & & & & & & & & & \\
\hline 53 & $\begin{array}{l}, 37 \\
6^{* *}\end{array}$ & $\begin{array}{l}17 \\
1^{* *}\end{array}$ & $\begin{array}{r}, 07 \\
8\end{array}$ & $\begin{array}{l}14 \\
2^{* *}\end{array}$ & $\begin{array}{l}, 23 \\
3^{* *}\end{array}$ & $\begin{array}{l}17 \\
2^{* *}\end{array}$ & $\begin{array}{l}, 73 \\
6^{* *}\end{array}$ & & & & & & & & & & & & \\
\hline 54 & $\begin{array}{l}, 35 \\
8^{* *}\end{array}$ & $\begin{array}{l}11 \\
5^{* *}\end{array}$ & $\begin{array}{r}, 07 \\
7\end{array}$ & $\begin{array}{r}10 \\
7^{*}\end{array}$ & $\begin{array}{l}18 \\
1^{* *}\end{array}$ & $\begin{array}{l}20 \\
2^{* *}\end{array}$ & $\begin{array}{l}76 \\
7^{* *}\end{array}$ & & & & & & & & & & & & \\
\hline 55 & $\begin{array}{l}, 35 \\
0^{* *}\end{array}$ & $\begin{array}{l}13 \\
5^{* *}\end{array}$ & $\begin{array}{r}, 09 \\
8^{*}\end{array}$ & $\begin{array}{l}13 \\
7^{* *}\end{array}$ & $\begin{array}{l}17 \\
7^{* *}\end{array}$ & $\begin{array}{l}19 \\
2^{* *}\end{array}$ & $\begin{array}{l}67 \\
0^{* *}\end{array}$ & & & & & & & & & & & & \\
\hline 56 & $\begin{array}{l}, 35 \\
4^{* *}\end{array}$ & $\begin{array}{l}16 \\
2^{* *}\end{array}$ & $\begin{array}{r}, 09 \\
7^{*}\end{array}$ & $\begin{array}{l}11 \\
4^{*}\end{array}$ & $\begin{array}{l}18 \\
6^{* *}\end{array}$ & $\begin{array}{l}16 \\
5^{* *}\end{array}$ & $\begin{array}{l}, 70 \\
1^{* *}\end{array}$ & & & & & & & & & & & & \\
\hline 57 & $\begin{array}{l}, 27 \\
0^{* *}\end{array}$ & $\begin{array}{r}, 08 \\
9^{*}\end{array}$ & $\begin{array}{r}, 03 \\
3\end{array}$ & $\begin{array}{r}, 07 \\
3\end{array}$ & $\begin{array}{r}10 \\
4^{*}\end{array}$ & $\begin{array}{l}15 \\
9^{* *}\end{array}$ & $\begin{array}{l}, 63 \\
2^{* *}\end{array}$ & & & & & & & & & & & & \\
\hline 58 & $\begin{array}{l}, 43 \\
8^{* *}\end{array}$ & $\begin{array}{l}, 33 \\
8^{* *}\end{array}$ & $\begin{array}{l}14 \\
6^{* *}\end{array}$ & $\begin{array}{l}, 29 \\
2^{* *}\end{array}$ & $\begin{array}{l}26 \\
8^{* *}\end{array}$ & $\begin{array}{l}27 \\
8^{* *}\end{array}$ & $\begin{array}{l}45 \\
0^{* *}\end{array}$ & & & & & & & & & & & & \\
\hline 59 & $\begin{array}{l}, 33 \\
0^{* *}\end{array}$ & $\begin{array}{l}16 \\
8^{* *}\end{array}$ & $\begin{array}{l}18 \\
1^{* *}\end{array}$ & $\begin{array}{l}19 \\
3^{* *}\end{array}$ & $\begin{array}{l}, 21 \\
0^{* *}\end{array}$ & $\begin{array}{l}10 \\
7^{*}\end{array}$ & $\begin{array}{l}45 \\
8^{* *}\end{array}$ & & & & & & & & & & & & \\
\hline
\end{tabular}




\begin{tabular}{rrrrrrrr}
60 &, 36 &, 16 &, 11 &, 13 &, 22 &, 21 &, 62 \\
& $3^{* *}$ & $2^{* *}$ & $3^{*}$ & $3^{* *}$ & $4^{* *}$ & $5^{* *}$ & $5^{* *}$ \\
61 &, 22 &, 08 &, 08 &, 11 &, 14 &, 11 &, 39 \\
& $9^{* *}$ & 0 & 1 & $1^{*}$ & $6^{* *}$ & $1^{*}$ & $9^{* *}$ \\
\hline
\end{tabular}

El instrumento evidencio una confiabilidad alta (Alfa $=0.88$ ), evaluada mediante el índice Alfa de Cronbach. Este mismo índice se calculó para cada una de las tres escalas que conforman el OSI, mostrando siempre una confiabilidad alta. Complementariamente, se realizó un análisis de máxima verosimilitud con rotación varimax con 14 factores con el ánimo de confirmar la agrupación de los ítems en cada factor con relación a la distribución original de los ítems en subescalas. La varianza total explicada fue de $43 \%$ y las cargas en su mayoría significativas oscilaron entre 0.14 y 0.87 (Ver Tabla 5).

Tabla 5.

Estructura factorial con rotación varimax

\begin{tabular}{|c|c|c|c|c|c|c|c|c|c|c|c|c|c|c|}
\hline \multirow[t]{2}{*}{ Ítem } & \multicolumn{6}{|c|}{ Roles Ocupacionales (ORQ) } & \multicolumn{4}{|c|}{ Tensión Personal (PSQ) } & \multicolumn{4}{|c|}{ Recursos Personales (PRQ) } \\
\hline & RO & RI & $\mathrm{RA}$ & RB & $\mathrm{R}$ & $\mathrm{PE}$ & VS & PSY & IS & PHS & $\mathrm{PRQ}$ & $\mathrm{RE}$ & SC & SS \\
\hline ORQ-1 & $\begin{array}{c}- \\
, 028\end{array}$ & & & & & & & & & & & & & \\
\hline ORQ-2 & 049 & & & & & & & & & & & & & \\
\hline ORQ-3 & ,095 & & & & & & & & & & & & & \\
\hline ORQ-4 & ,190 & & & & & & & & & & & & & \\
\hline ORQ-5 &, 253 & & & & & & & & & & & & & \\
\hline ORQ-6 & ,110 & & & & & & & & & & & & & \\
\hline ORQ-7 & $\begin{array}{c}- \\
, 021\end{array}$ & & & & & & & & & & & & & \\
\hline ORQ-8 & ,091 & & & & & & & & & & & & & \\
\hline ORQ-9 & $\begin{array}{c}- \\
, 111\end{array}$ & & & & & & & & & & & & & \\
\hline ORQ-10 &, 052 & & & & & & & & & & & & & \\
\hline ORQ-11 & & ,732 & & & & & & & & & & & & \\
\hline ORQ-12 & & ,739 & & & & & & & & & & & & \\
\hline ORQ-13 & & ,672 & & & & & & & & & & & & \\
\hline ORQ-14 & & ,403 & & & & & & & & & & & & \\
\hline ORQ-15 & & ,614 & & & & & & & & & & & & \\
\hline ORQ-16 & & ,787 & & & & & & & & & & & & \\
\hline ORQ-17 & & ,783 & & & & & & & & & & & & \\
\hline ORQ-18 & & ,715 & & & & & & & & & & & & \\
\hline ORQ-19 & & ,260 & & & & & & & & & & & & \\
\hline ORQ-20 & & ,658 & & & & & & & & & & & & \\
\hline ORQ-21 & & ,310 & & & & & & & & & & & & \\
\hline ORQ-22 & & & ,070 & & & & & & & & & & & \\
\hline ORQ-23 & & &, 523 & & & & & & & & & & & \\
\hline ORQ-24 & & & ,313 & & & & & & & & & & & \\
\hline ORQ-25 & & &, 585 & & & & & & & & & & & \\
\hline ORQ-26 & & &, 555 & & & & & & & & & & & \\
\hline ORQ-27 & & & ,055 & & & & & & & & & & & \\
\hline ORQ-28 & & & ,393 & & & & & & & & & & & \\
\hline ORQ-29 & & &, 561 & & & & & & & & & & & \\
\hline ORQ-30 & & & ,335 & & & & & & & & & & & \\
\hline ORQ-31 & & & 173 & & & & & & & & & & & \\
\hline
\end{tabular}




\section{Mónica García Rubiano, Helena Vélez Botero}

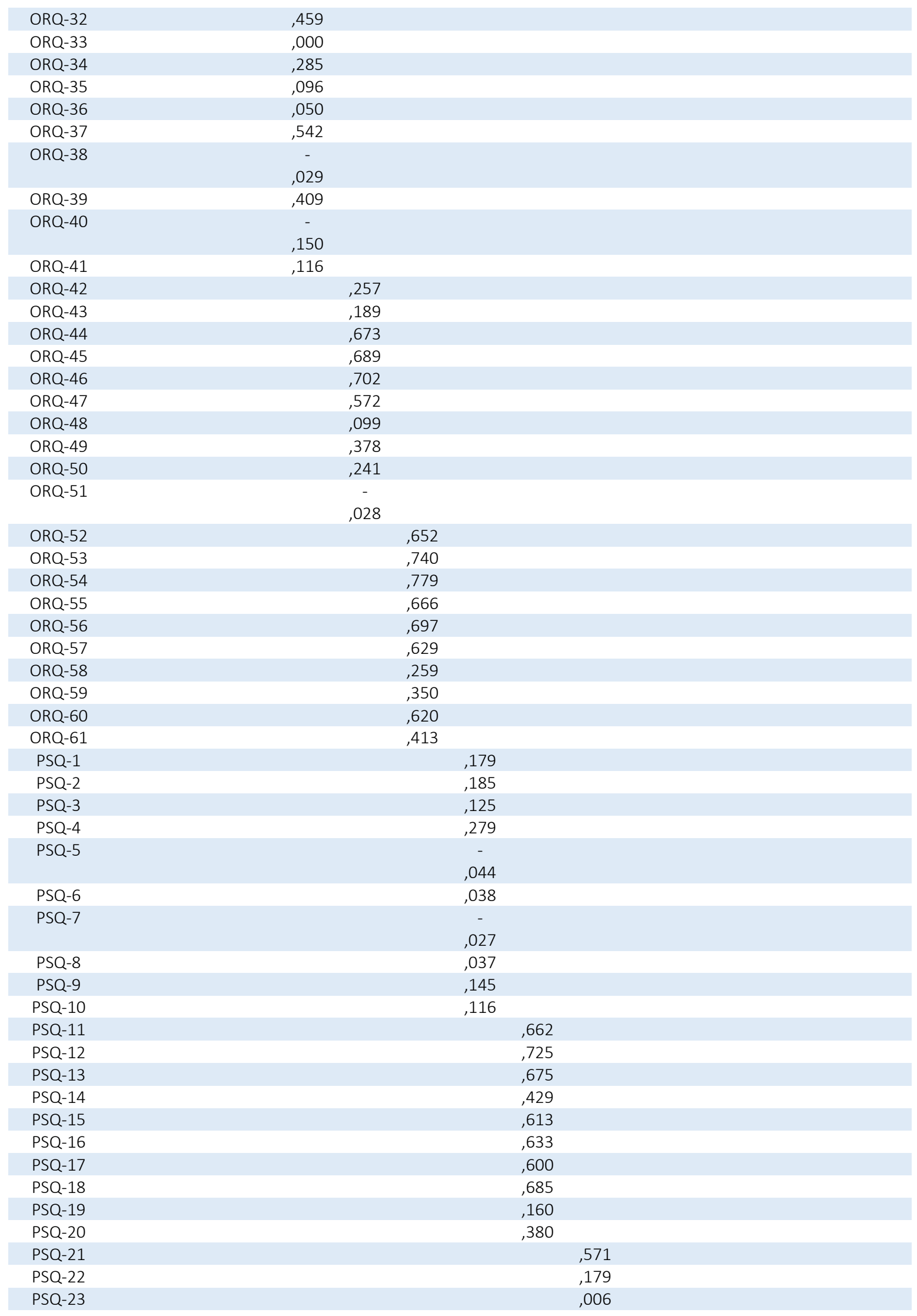




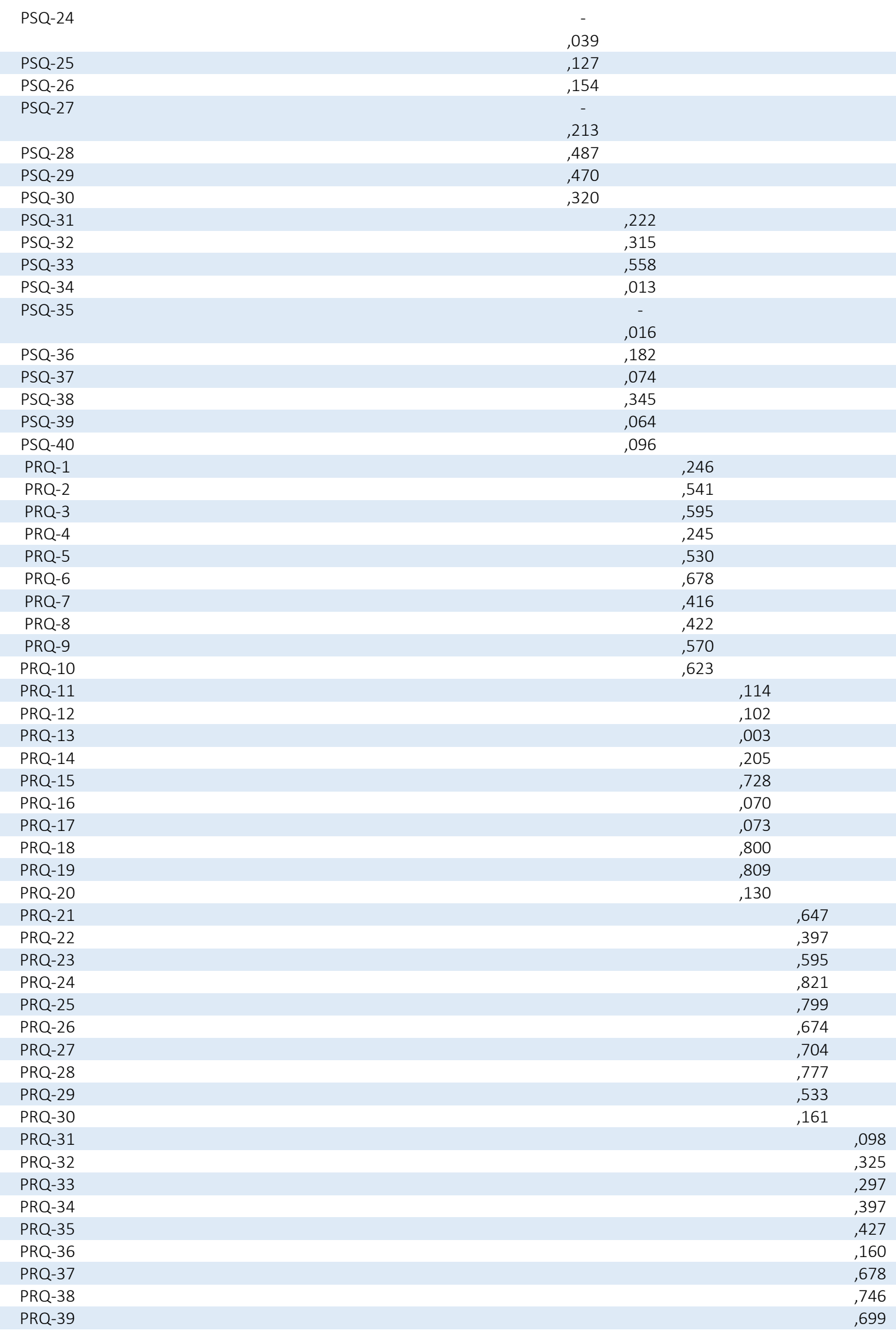




\section{Mónica García Rubiano, Helena Vélez Botero}

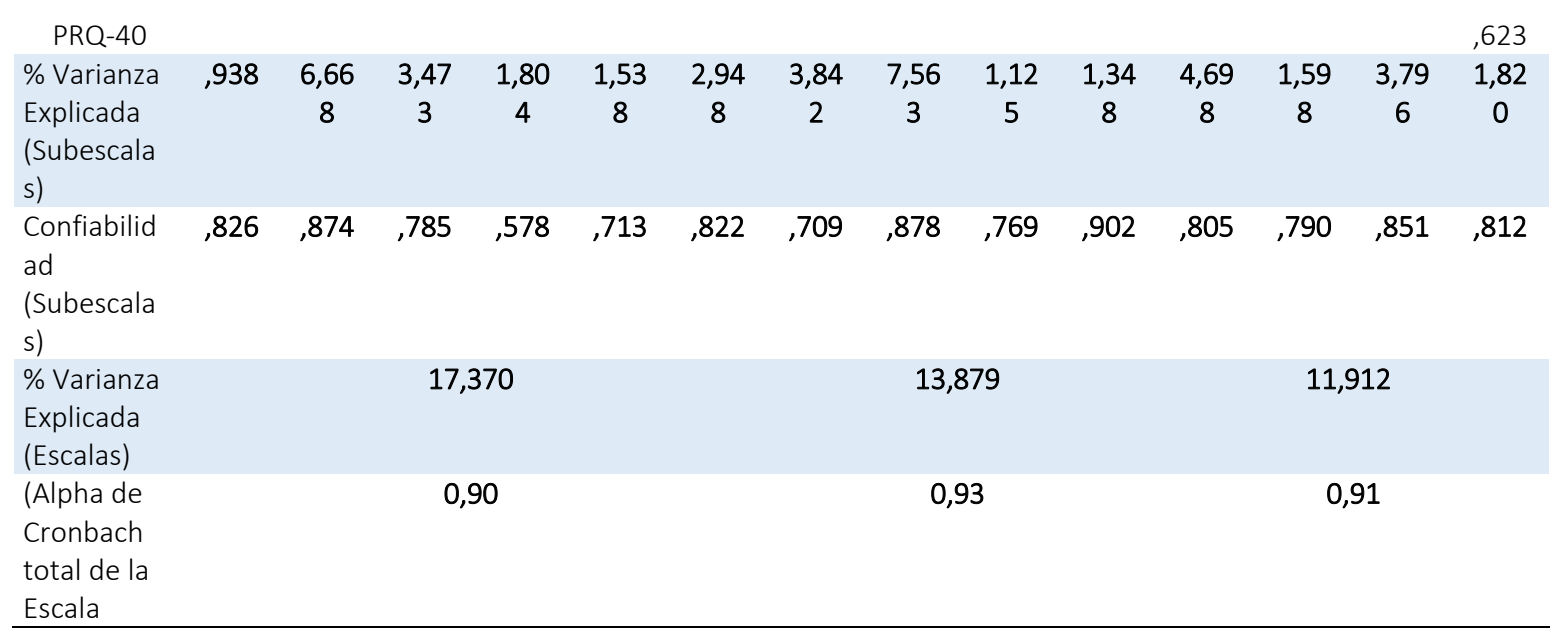

\section{DISCUSIÓN}

El objetivo de este estudio fue explorar las características psicométricas del inventario de estrés ocupacional (OSI), con el ánimo de identificar si se trata de una herramienta con propiedades adecuadas para la evaluación del estrés en la población trabajadora colombiana.

En primer lugar hay que mencionar la prueba $t$, cuyos resultados evidencian una adecuada capacidad discriminativa de los ítems en tanto que la significancia estadística es menor a 0,05 para todos los ítems y los valores de las desviaciones típicas son en general altos. De este análisis es posible concluir que los ítems que componen el inventario de estrés ocupacional-OSI poseen una adecuada capacidad discriminativa, siendo sensibles a las diferencias individuales en la manifestación de estrés.

Por su parte, el análisis del coeficiente alfa de Cronbach mostró una confiabilidad alta para el instrumento en general $(0,88)$. Para el caso de las escalas que componen el instrumento se encontró una confiabilidad de 0,90 para la escala "roles ocupacionales", 0.93para la escala "tensión personal" y 0,91 para la escala "recursos personales". Estos hallazgos son consistentes con las propiedades reportadas por Schmidt, et al. (2003). Adicionalmente, este análisis evidencia que la eliminación de cualquiera de los reactivos no aportaría de forma significativa al mejoramiento del coeficiente de confidencialidad, por lo que se asume que el contenido de los ítems contempla aspectos relevantes en la medición del estrés y los constructos contemplados en el cuestionario (Hancock \& Mueller, 2010).

Estos datos son comparables con el estudio de Jackson (2004), quien realizó un estudio comparativo del inventario original del OSI (Osipow \& Spokane, 1987) con el OSI-R (Osipow, 1998), los resultados evidencian que la confiabilidad general del instrumento es de 0,88 . Para las escalas que componen el instrumento la confiabilidad es de 0,93 (roles ocupacionales), tensión personal $(0,89$ ) y entre 0,70 0,89 para recursos personales.

Ahora bien, los resultados de las correlaciones entre escalas, subescalas e ítems complementan este aná- lisis de confiabilidad-ítem e ítem-ítem. Las correlaciones entre escalas y subescalas evidencian una asociación significativa alta entre cada escala y las subescalas que la componen. Es preciso 
mencionar que las subescalas que componen la escala "recursos personales" se correlacionan de forma negativa y significativa con las otras escalas del instrumento, poniendo de manifiesto que en presencia de recursos para afrontar el estrés, las puntuaciones sobre el conflicto que genera el rol ocupacional y las manifestaciones de tensión serán menores (Arias, 2012).

Los coeficientes de correlación entre escalas, subescalas y los ítems que las conforman presentan de manera común correlaciones positivas y significativas, evidenciando una adecuada construcción de las escalasy subescalas en relación con sus contenidos; lo cual a su vez es un indicador de la pertinencia de cada grupo de ítems para evaluar los constructos asociados a la evaluación del estrés. El estudio realizado por Jackson (2004), evidencia que los coeficientes de correlación de los componentes del inventario fueron iguales o mayores a 0,63 y todos estadísticamente significativos. Concluye el autor que las correlaciones relativamente altas entre el OSI y el OSI-R son similares como para la generalización de la validez del inventario.

Finalmente, el análisis factorial confirmatorio, muestra que el inventario tiene la capacidad para explicar el 43 \% de la varianza en el constructo "estrés" a partir de los 14 componentes (subescalas) definidos en la estructura del instrumento. Sin embargo, se evidencia que la agrupación de los ítems en los componentes resulta algo errática y no permite corroborar su distribución de acuerdo con la estructura original del cuestionario; lo cual señala la necesidad de repetir este ejercicio y ampliar el tamaño de la muestra para poseer información suficiente en cuanto a la conformación de los factores (Lloret-Segura, Ferreres-Traver, Hernández-Baeza \& Tomás-Marco, 2014).

Con base en la exploración realizada, es posible afirmar que los resultados obtenidos señalan al inventario de estrés ocupacional-OSI como un instrumento con propiedades psicométricas consistentes con lo reportado en la adaptación argentina del instrumento, haciendo de este una herramienta de medición potencialmente adecuada para la población colombiana. Esto teniendo en cuenta que el inventario de estrés ocupacional-OSI: a) muestra niveles favorables de discriminación, b) tiene una estructura armónica evidente en las asociaciones entre sus componentes (escalas, subescalas e ítems) y, c) posee una confiabilidad alta (Bandalos \& Finney, 2010).

Así pues, esta primera aproximación, presenta al inventario de estrés ocupacional-OSI como un instrumento de medida confiable y sensible, con la capacidad para evaluar de forma cabal el estrés en la población trabajadora colombiana. De tal suerte que resulta de suma importancia darle continuidad a este estudio, no solo para ahondar en las condiciones de validez del instrumento, sino también para establecer de forma precisa los criterios de baremación requeridos para la interpretación de sus resultados en el contexto colombiano.

Nota de Autores

El artículo es derivado de la investigación: Exploración de las características psicométricas del inventario de estrés ocupacional 


\section{Mónica García Rubiano, Helena Vélez Botero}

\section{REFERENCIAS}

Aguirre, A. \& Quijano, A. (2015). Síndrome por quemarse en el trabajo y variables familiares y laborales de los médicos generales de Bogotá. Una estrategia de calidad laboral. Revista Colombiana de Psiquiatría, 44(4), 198-205. DOI: https://doi.org/10.1016/j.rcp.2015.05.017

Arce, S. (2012). Factores organizacionales causantes del estrés en el trabajo y estrategias para afrontarlo. Revista Venezolana de Gerencia, 17(60), 611-634. Dsiponible en http://produccioncientificaluz.org/index.php/rvg/article/view/10935

Arias, G. (2012). Estrés laboral en trabajadores desde el enfoque de los sucesos vitales. Revista Cubana de Salud Pública, 38(4), 2-30. Disponible en http://bvs.sld.cu/revistas/spu/vol38_4_12/spu04412.htm

Bandalos, D.L. \& Finney, S.J. (2010). Factor Analysis: Exploratory and Confirmatory. In G.R. Hancock \& R.O. Mueller (Eds.), Reviewer's guide to quantitative methods. New York: Routledge.

Beh, L. \& Loo, L. (2012). Job Stress and Coping Mechanisms among Nursing Staff in Public Health Services. International Journal of Academic Research in Business and Social Sciences, 2(7), 131-176. Dsiponible en http://www.hrmars.com/admin/pics/912.pdf

Carracedo-Martínez, E. \& Figueiras, A. (2006). Tratamiento estadístico de la falta de respuesta en estudios epidemiológicos transversales. Revista de Salud Pública de México, 48(4), 341-347. Dsiponible en http://www.scielo.org.mx/scielo.php?script=sci_arttext\&pid=S0036-36342006000400009

Carretero-Dios, H. \& Pérez, C. (2007). Normas para el desarrollo y revisión de estudios instrumentales: consideraciones sobre la selección de tests en la investigación psicológica. International Journal of Clinical and Health Psychology, 7(3), 863-882. Dsiponible en http://aepc.es/ijchp/NDREI07_es.pdf

Castillo, I., Torres, N., Ahumada, A., Cardenas, K. \& Licona, S. (2014). Estrés laboral en enfermería y factores asociados. Cartagena (Colombia). Revista Salud Uninorte, 30(1), 34-43. Dsiponible en http://rcientificas.uninorte.edu.co/index.php/salud/article/viewArticle/5333

Charter, R.A. (2003). A breakdown of reliability coefficients by test type and reliability method, and the clinical implications of low reliability. Journal of General Psychology, 130(3), $290-304$. http://dx.doi.org/10.1080/00221300309601160

Cirera, Y., Aparecida, E., Rueda, V. \& Ferraz, O. (2012). Impacto de los estresores laborales en los profesionales y en las organizaciones. Análisis de investigaciones publicadas. Invenio, 15(29), 67-80. Dsiponible en http://vufind.uniovi.es/Record/oai:doaj.orgarticle:680a17663ffd40eca84b3fb3bcd1489d/Details

Dahl, M.S. (2010). Organizational change and employee stress. Management Science, 57(2), $240-256$. https://doi.org/10.1287/mnsc.1100.1273

García-Neira, M.C., Piraquive, K.G. \& Salazar, L.J. (2012). Validez de contenido del cuestionario de estrés ocupacional (OSI) en la ciudad de Bogotá, Colombia. (Trabajo de grado). Universidad Santo Tomás, Colombia. Disponible en

http://repository.usta.edu.co/bitstream/handle/11634/3252/Garciamaria2012.pdf?sequence=1\&isAllowed= y 


\section{Mónica García Rubiano, Helena Vélez Botero}

Hancock, G.R. \& Mueller, O. (2010). The Reviewer's Guide to Quantitative Methods in the Social Sciences. (3rd Ed.). New York, NY: Routledge.

Hermosa, A.M. \& Perilla, L.E. (2015) Retos investigativos en psicología de la salud ocupacional: el estrés laboral. Rev. Fac. Nac. Salud Pública 5, 3(2), 252- 261. DOI: 10.17533/udea.rfnsp.v33n2a12

Hernández, S., Fernández, C. \& Baptista, P. (2006). Metodología de la investigación. México: McGraw-Hill.

Hicks, R. E., Sabanci, A. \& Bahr, M. (2015). Assessing Stress at Work across Occupations and Cultures Using the Occupational Stress Inventory Revised. World Journal of Management, 6(1), 1-9. Dsiponible en http://epublications.bond.edu.au/fsd_papers/88/

Jackson, A. (2004). A Survey of the Occupational Stress, Psychological Strain, and Coping Resources of Licensed Professional Counselors in Virginia: A Replication Study. (Tesis doctoral). Virginia Polytechnic Institute, Estados Unidos. Dsiponible en https://vtechworks.lib.vt.edu/handle/10919/30206

Lazarus, R. \& Folkman, S. (1986). Estrés y procesos cognitivos. Barcelona: Martínez Roca.

Leibovich de Figueroa, N. \& Schmidt, V. (2004). El uso de instrumentos psicológicos para la evaluación del estrés ocupacional en nuestro medio. Revista Iberoamericana de Diagnóstico y Evaluación Psicológica, 17(1), 139157. Dsiponible en http://www.aidep.org/03_ridep/R17/R177.pdf

López, L. \& Campos, J. (2002). Evaluación de Factores presentes en el Estrés Laboral. Revista de Psicología, 11(1), 149165. DOI: 10.5354/0719- 0581.2002.17282

Lloret-Segura, S., Ferreres-Traver, A., Hernández-Baeza, A. \& Tomás-Marco, I. (2014). El análisis factorial exploratorio de los ítems: una guía práctica, revisada y actualizada. Anales de Psicología, 30(3), 1151- 1169. DOI: 10.6018/analesps.30.3.199361

Magaya, L., Asner-Self, K. \& Schreiber, J. (2005). Stress and coping strategies among Zimbabwean adolescents. British Journal of Educational Psychology, 75(4), 661-671. DOI: 10.1348/000709905X25508

Merín-Reig, J., Cano-Vindel, A. \& Tobal, J.J. (1995). El estrés laboral: bases teóricas y marco de intervención. Ansidad y Estrés, 1(2-3), 113-130. Disponible en https://dialnet.unirioja.es/servlet/articulo?codigo=4195267

Merino-Soto, C. (2016). Percepción de la claridad de los ítems: Comparación del juicio de estudiantes y jueces-expertos. Revista Latinoamericana de Ciencias Sociales, Niñez y Juventud, 14(2), 1469-1477. DOI: 10.11600/1692715x.14239120615

Merino-Soto, C. \& Segovia, L. (2009). Intervalos de confianza asimétricos para el índice de validez de contenido: Un programa Visual Basic para la V de Aiken. Anales de psicología, 25(1), 169-171. Dsiponible en https://dialnet.unirioja.es/servlet/articulo?codigo=2973711

Osipow, S. H. (1998). A manual for the Occupational Stress Inventory Revised Edition (Professional Manual). Luz, Florida: Psychological Assessment Resources.

Osipow, S.H. \& Spokane, A.R. (1987). A manual for the occupational stress inventory (Research Version). Odessa, FL: Psychological Assessment Resources.

Padilla, J., García, A. \& Gómez, J. (2007). Cuestionarios mediante procedimientos. Avances en medición, 5, 115-126. Dsiponible 


\section{Mónica García Rubiano, Helena Vélez Botero}

http://www.humanas.unal.edu.co/psicometria/files/8813/7036/5473/Evaluacin_De_Cuestionarios_Mediant e_Procesos_Cognitivos.pdf

Peiró, J.M. (2009). Nuevas tendencias en la investigación sobre estrés laboral y sus implicaciones para el análisis y prevención de los riesgos psicosociales. Instituto Valenciano de Investigaciones Económicas (IVIE). Disponible en http://web2011.ivie.es/downloads/2009/09/Leccion_magistral_JMPeiro.pdf

Peiró, J.M. \& Rodríguez, I. (2008). Estrés laboral, liderazgo y salud organizacional. Papeles del psicólogo, 29(1), 68-82.

Ministerio de la Protección Social (2008). Res. 2646. 47059 (legislado). Disponible en http://www.papelesdelpsicologo.es/pdf/1540.pdf

Ramos, A., Moreno, P. \& Ales, Y. (2015). Estrés de rol y satisfacción laboral: examinando el papel mediador del engagement en el trabajo. Revista de Psicología del Trabajo y de las Organizaciones. 31(2), 69-77.: https://doi.org/10.1016/j.rpto.2015.04.001

Rodríguez, R. \& De Rivas, S. (2011). Los procesos de estrés laboral y desgaste profesional (burnout): diferenciación, actualización y líneas de intervención. Medicina y seguridad del trabajo, 57(1), 72-88. http://dx.doi.org/10.4321/S0465-546X2011000500006.

Sánchez, F.C. (2011). Estrés laboral, satisfacción en el trabajo y bienestar psicológico en trabajadores de una industria cerealera. Bogotá: Universidad Abierta Interamericana. Disponible en http://imgbiblio.vaneduc.edu.ar/fulltext/files/TC111836.pdf

Schmidt, V., Leibovich de Figueroa, N., Schufer, M., González, M. \& Marconi, A. (2003). Inventario de estrés ocupacional (OSI): la muestra argentina. Buenos Aires, Argentina: Departamento de Publicaciones, Facultad de Psicología, U. B. A.

Schneiderman, N., Ironson, G. \& Siegel, S. (2005). Stress and Health: Psychological, Behavioral and Biological Determinants. The Annual Review of Clinical Psychology; 1, 607-628. DOI: 10.1146/annurev.clinpsy.1.102803.144141

Walter, L. \& Arias, G. (2012). Estrés laboral en trabajadores desde el enfoque de los sucesos vitales. Revista Cubana de la Salud Pública, 38(4), 525-535. Dsiponible en http://bvs.sld.cu/revistas/spu/vol38_4_12/spu04412.htm 